\title{
The Nrf2-NLRP3-caspase-1 axis mediates the neuroprotective effects of Celastrol in Parkinson's disease
}

\author{
Chenyu Zhang ${ }^{\text {a,1 }}$, Miao Zhao ${ }^{\text {a,1 }}$, Bingwei Wang ${ }^{\text {a }}$, Zhijie Su ${ }^{\text {a }}$, Bingbing Guo ${ }^{\text {a }}$, Lihua Qin ${ }^{\text {a }}$ \\ Weiguang Zhang ${ }^{\mathrm{a}}$, Ruimao Zheng ${ }^{\mathrm{a}, \mathrm{b}, \mathrm{c}, \mathrm{d}, \text {, }}$ \\ ${ }^{\text {a }}$ Department of Anatomy, Histology and Embryology, Health Science Center, Peking University, Beijing, China \\ ${ }^{\mathrm{b}}$ Neuroscience Research Institute, Peking University, Beijing, China \\ ${ }^{\mathrm{c}}$ Key Laboratory for Neuroscience of Ministry of Education, Peking University, Beijing, China \\ ${ }^{\mathrm{d}}$ Key Laboratory for Neuroscience of National Health Commission, Peking University, Beijing, China
}

\section{A R T I C L E I N F O}

\section{Keywords:}

Parkinson's disease

Celastrol

Nrf2

NLRP3

Caspase-1

$\alpha$-synuclein

\begin{abstract}
A B S T R A C T
Parkinson's disease (PD) is a chronic neurodegenerative disorder that is characterized by motor symptoms as a result of a loss of dopaminergic neurons in the substantia nigra pars compacta (SNc), accompanied by chronic neuroinflammation, oxidative stress, formation of $\alpha$-synuclein aggregates. Celastrol, a potent anti-inflammatory and anti-oxidative pentacyclic triterpene, has emerged as a neuroprotective agent. However, the mechanisms by which celastrol is neuroprotective in PD remain elusive. Here we show that celastrol protects against dopamine neuron loss, mitigates neuroinflammation, and relieves motor deficits in MPTP-induced PD mouse model and AAV-mediated human $\alpha$-synuclein overexpression PD model. Whole-genome deep sequencing analysis revealed that Nrf2, NLRP3 and caspase-1 in SNc may be associated with the neuroprotective actions of celastrol in PD. By using multiple genetically modified mice (Nrf2-KO, NLRP3-KO and Caspase-1-KO), we identified that celastrol inhibits NLRP3 inflammasome activation, relieves motor deficits and nigrostriatal dopaminergic degeneration through Nrf2-NLRP3-caspase-1 pathway. Taken together, these findings suggest that Nrf2-NLRP3-caspase-1 axis may serve as a key target of celastrol in PD treatment, and highlight the favorable properties of celastrol for neuroprotection, making celastrol as a promising disease-modifying agent for PD.
\end{abstract}

\section{Introduction}

Parkinson's disease (PD) is the second most common neurodegenerative disease worldwide, affecting about $2 \%$ of the population over the age of 60 , and the incidence of PD is expected to increase with the aging of the population $[1,2]$. The pathological hallmark of PD is the progressive loss of dopaminergic neurons in substantia nigra pars compacta (SNc), accompanied with the accumulation of misfolded $\alpha$-synuclein ( $\alpha$-syn) in Lewy bodies, which decreases striatal dopamine, and thus leads to motor dysfunction, including resting tremors, bradykinesia and rigidity [2-4]. Although the pathology of PD remains unclear, compelling evidence from clinical, preclinical, and epidemiological studies suggests that neuroinflammation and oxidative stress may play central roles in PD pathogenesis $[3,5,6]$. To date, despite substantial efforts, there is still no disease-modifying agent to prevent the progression of PD; and gene therapy clinical trials for PD also failed to halt or slow the disease process of PD [7-11]. Therefore, there's an urgent need to discover new therapeutic agents to protect against the loss of dopaminergic neurons in PD. In light of this, the development of promising agents exhibiting disease-modifying properties is critical for PD treatment.

Notably, natural products show a promising role in the development of novel agents for PD treatment due to their pharmacological properties linked to neuroprotection [12-14]. Celastrol, a pentacyclic triterpene, is an active component of tripterygium wilfordi (thunder god vine). Celastrol has shown favorable neuroprotective properties in neurodegenerative diseases because of its anti-oxidative and anti-inflammatory effects [15-17]. Celastrol protects against the neurotoxin-induced loss of dopaminergic neurons $[15,18]$, suppresses the activation of microglia [16], reduces the production of neuroinflammatory cytokines [16], and inhibits the nitric oxide generation induced by inducible nitric oxide synthase (iNOS) [16,19,20]. Celastrol promotes autophagy [15],

\footnotetext{
* Corresponding author. Department of Anatomy, Histology and Embryology, Health Science Center, Peking University, Beijing, China.

E-mail address: rmzheng@pku.edu.cn (R. Zheng).

1 These authors contributed equally: Chenyu Zhang, Miao Zhao.
} 
ameliorates the mitochondrial dysfunction [21], and inhibits lipid peroxidation induced by $\mathrm{Fe}^{2+}$ [22]. Intriguingly, the correspondences between celastrol effects and PD pathogenesis raise the possibility that celastrol might be a promising candidate for treatment of PD. However, the mechanism underlying the neuroprotective action of celastrol remains elusive.

To investigate the potential mechanisms underlying the neuroprotective effects of celastrol in PD, we initially performed wholegenome deep sequencing analysis to explore the differential expression genes (DEGs) in the SNc of celastrol-treated PD mouse model. This unbiased high-throughput approach enables the identification of the genes associated with the neuroprotective effects of celastrol, such as Nrf2, HO1, Nlrp3, Caspase-1 and ASC. Nrf2 (nuclear-factor-E2-related factor 2), a key transcription factor, plays a critical role in PD pathogenesis [23]. Under homeostatic conditions, Nrf2 resides in cytosol and is degraded by ubiquitination and proteolysis. Under the condition of PD-causing toxins, Nrf2 translocates to the nucleus where it binds to antioxidant response element (ARE) to induce antioxidant and phase II detoxification enzymes, and thus exhibits anti-oxidative and anti-inflammatory effects [24-26]. Human genetic studies show that a functional haplotype in the Nrf2 gene promoter, confers high transcriptional activity of Nrf2 and decreased incidence of PD in Swedish and Polish [27,28]. Selegiline (deprenyl), a type-B monoamine oxidase (MAO-B) inhibitor, which is currently used in PD treatment, also displays anti-oxidative effect via Nrf2 [29]. Overexpression of Nrf2 protects against motor pathology and $\alpha$-synuclein aggregation [30]; whereas deficiency of Nrf2 exacerbates PD phenotypes in mice [24,27]. Celastrol relieves inflammation in diet-induced obese mice via Nrf2 pathway [31]. Nevertheless, whether the neuroprotective action of celastrol is mediated by Nrf2 remains unknown.

NLRP3 (NOD-, LRR- and pyrin domain-containing protein 3), a cytosolic protein, belongs to critical inflammatory signaling complex called inflammasome, which participates in the pathogenesis of PD [2, 32]. The NLRP3 inflammasome is a multiprotein complex that acts as intracellular sensor of environmental and cellular stress, which is composed of NLRP3, apoptosis associated speck-like protein containing a caspase recruitment domain (ASC) and caspase-1 protease [2]. The activation of NLRP3 promotes the secretion of IL1 $\beta$ and induces pyroptosis [33]. Activation of NLRP3 inflammasome has been observed in the SNc of PD patients [2]. Histologic studies reveal elevated NLRP3 expression in mesencephalic neurons of PD patients, and show a rare NLRP3 polymorphism associated with decreased risk of PD [34]. The neurotoxins, oxidative stress, aggregation of $\alpha$-synuclein, genetic mutations, and bacterial toxins can activate the NLRP3 inflammasome, and trigger caspase- 1 activation as well as the caspase-1-mediated release of IL1 $\beta$ and IL18, leading to the death of dopaminergic neurons in the SNc [2,32,35-37]. A novel Nrf2 activator (DDO7263) targeting brain tissue, protects against MPTP-induced PD model by suppressing the NLRP3 inflammasome and oxidative stress [38]. Astragaloside IV also ameliorates MPTP-induced dopamine neuron loss and motor deficits through suppression of NLRP3 inflammasome [39]. Celastrol reduces the secretion of IL1 $\beta$ and IL18 by inhibiting both NLRP3 expression and cleavage of caspase-1 [40]. These observations indicate that the activated Nrf2 suppresses NLRP3 to mitigate the neuroinflammation. However, whether the celastrol inhibits NLRP3 inflammasome through Nrf2 to exert neuroprotective effects in PD is not yet known.

To assess the neuroprotective actions of celastrol in PD, we employed multiple PD models to better understand the nigro-striatal degeneration, including MPTP-induced PD mouse model and the AAV-mediated human $\alpha$-syn overexpression PD model. By using genomewide RNAsequencing approach and the genetically modified mice (Nrf2-KO, NLRP3-KO and Caspase-1-KO mice), we identified that Nrf2 and NLRP3 play key roles in the neuroprotective effects of celastrol. Altogether, we provide a direct evidence that celastrol protects against loss of dopaminergic neurons, maintains nigro-striatal system function, and mitigates neuroinflammation by suppressing NLRP3 inflammasome via activation of Nrf2. These findings suggest that the activation of NLRP3 might be a key pathological factor that drives PD pathogenesis; whereas the suppression of NLRP3 through the Nrf2 pathway may underlie the neuroprotective effects of celastrol, which renders celastrol as a promising disease-modifying agent to alleviate progressive dopaminergic degeneration.

\section{Results}

\subsection{Celastrol protects against loss of dopaminergic neurons in $S N c$ of MPTP-induced PD mice}

To assess the neuroprotective potential of celastrol in PD, the MPTPinduced PD mouse model was firstly employed. It is reported that celastrol may exhibit biphasic pharmacological properties. Celastrol at low doses protects against the neurotoxicity; while celastrol at high doses exacerbates the neurotoxicity [41]. Therefore, to gain the desired therapeutic outcome of celastrol, we examined a range of dosages (Supplementary Fig. 1-3) and treatment course (Supplementary Fig. 4-6), and finally we decided to initiate treatment with celastrol (10 $\mu \mathrm{g} / \mathrm{kg}$ ) for 7 days in MPTP-induced PD mouse model to explore the mechanism underlying the neuroprotective effects of celastrol, as shown in Fig. 1a. Celastrol treatment led to a marked restoration of the loss of dopaminergic neurons ( $\sim 70 \%$ vs. $48 \%, P<0.01$ ) (Fig. 1b), rescued the reduction of TH protein levels in the SNc $(\sim 66 \%$ vs. $47 \%, P<0.001)$ (Fig. 1c) and STR (70\% vs. 48\%, $P<0.05$ ) (Supplementary Fig. 8b). The decrease of $\mathrm{TH}^{+}$fibers density $(\sim 74 \%$ vs. $50 \%, P<0.01)$ was also mitigated by celastrol (Supplementary Fig. 8a). The pole descent, rotarod test, beam traversal, hindlimb clasping reflexes, and gait test showed that the impaired motor coordination and balance were relieved by celastrol (Fig. $1 \mathrm{~d}$ and e). Together, these results suggest that celastrol restores the dopamine synthesis, maintains the nigrostriatal function, and normalizes the motor function in the MPTP-induced PD mice.

Given that celastrol exhibits profound anti-neuroinflammatory effects [16,17], the effects of celastrol on neuroinflammation triggered by MPTP was assessed. We observed that celastrol treatment led to decreased number of $\mathrm{GFAP}^{+}$(glial fibrillary acidic protein) and $\mathrm{Iba}^{+}$ (ionized calcium-binding adapter molecule 1) cells, as well as GFAP and Iba1 protein levels in both SNc and STR (Supplementary Fig. 9a-c), suggesting that celastrol may mitigate the reactive glial cell-mediated neuroinflammation. Similarly, quantitative PCR analysis showed that celastrol suppressed the expression of the genes associated with pro-inflammatory (TNF $\alpha, I L 1, I L 6)$, while activated the genes linked to anti-inflammatory (IL4, IL10) (Fig. 1f). Celastrol led to an enhancement of the genes related to anti-oxidative stress (Nrf2, HO1, NQO1, GCLC and GCLM), mitochondrial function (Pgc1 $\alpha, U c p 2, \operatorname{Drp} 1$ and $M f n 1)$; and normalized the genes linked to apoptosis (Caspase 3 and Caspase9) and ER stress (CHOP, GRP78, ERN1, Xbp1 and ATF6) (Fig. 1f), highlighting the multiple bioactivities of celastrol in PD. Collectively, these results demonstrate that celastrol can relieve the MPTP-induced PD-like symptoms, rendering celastrol as a promising disease-modifying agent of PD.

\subsection{Nrf2, NLRP3 and Caspase-1 are identified as therapeutic targets of celastrol}

To explore the mechanism underlying the neuroprotective action of celastrol in PD, whole-genome RNA-sequencing analysis was performed. The differential expression genes (DEGs) in the SNc of MPTP-induced PD mice treated with celastrol were identified. Gene expression profiles (Fig. 2a) and DEGs (Fig. 2b) were visualized as heatmap respectively. Celastrol restored the decreased expression of the genes linked to locomotory behavior (TH, SLC6A3, Nr4a2, Drd2 and Trpc2); anti-oxidative stress (Nfe2l2, Hmox1, Nqo1, Sod2, Gpx1); autophagy-lysosomal pathway (Beclin1, Ern1 and Hspa5) and ubiquitin-proteasome system (Usp14, Alfy, Hsp70) under the condition of MPTP treatment (Fig. 2a and 
a

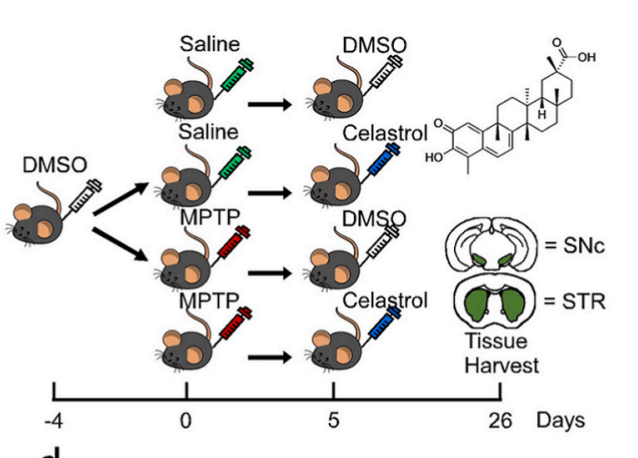

d
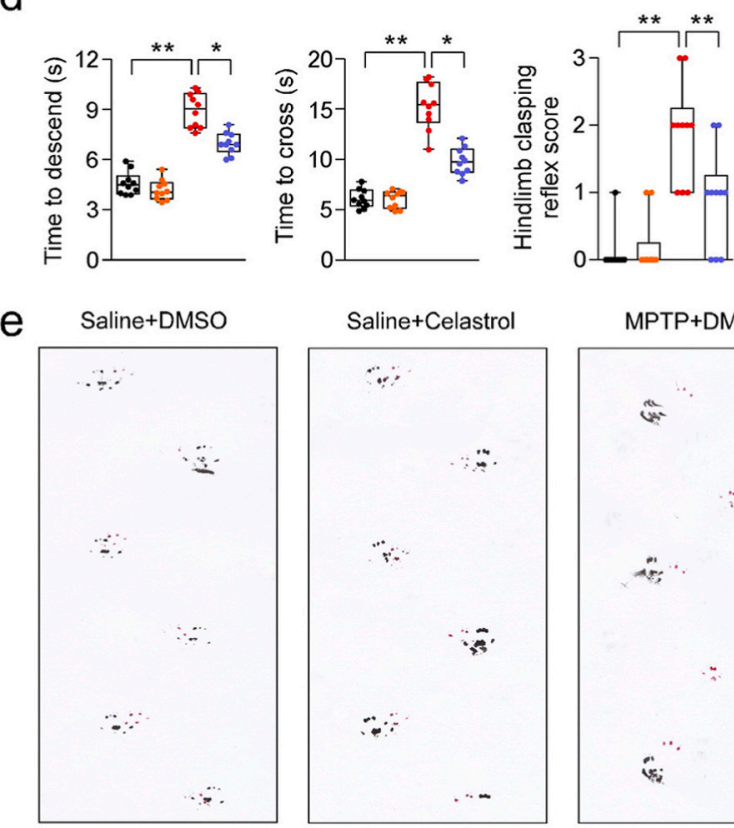

b

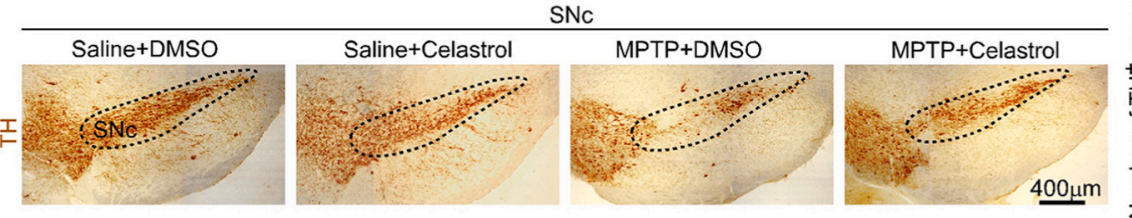

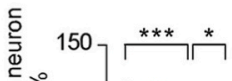

- Saline+DMSO

亭. Saline+Celastrol I. MPTP+Celastrol

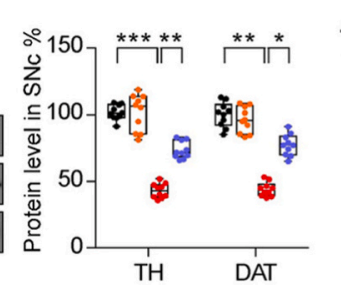

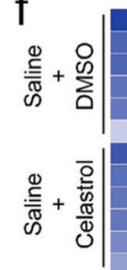

$\frac{0}{\sum}+\frac{0}{2}+$

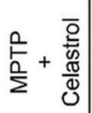

High

|
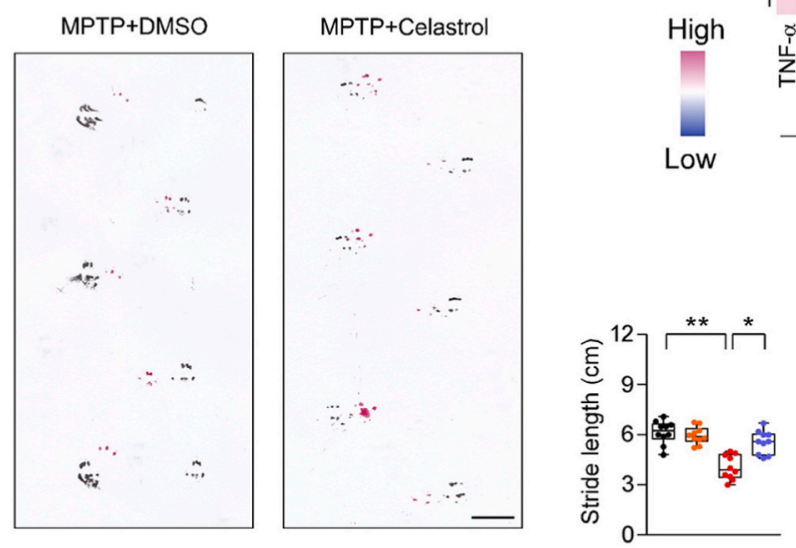

管
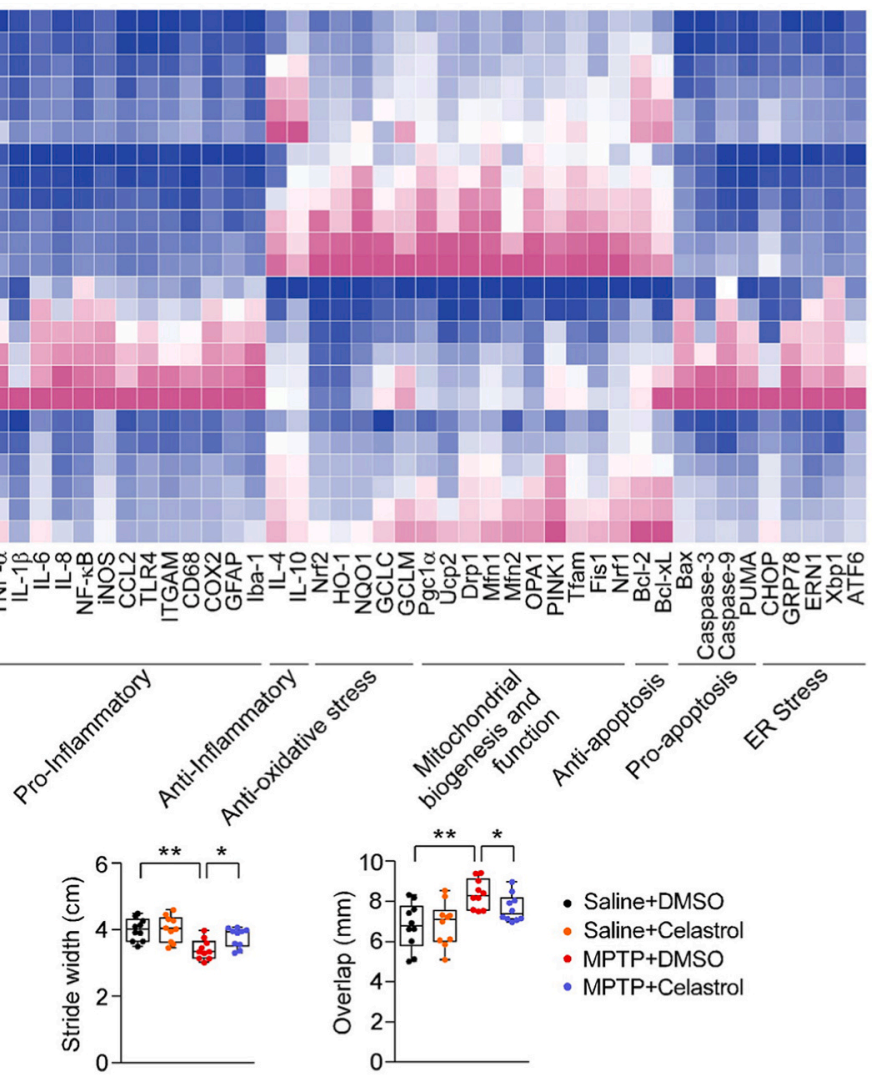

है

- Saline+DMSO

- MPTP+DMSO

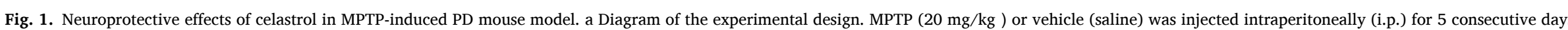

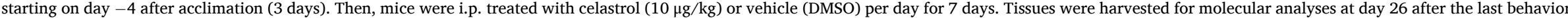

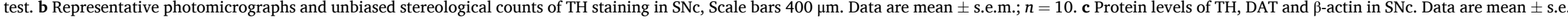

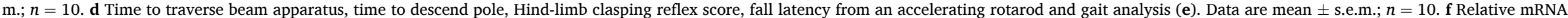

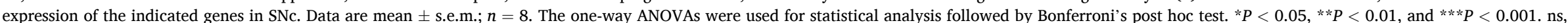
not significant. 


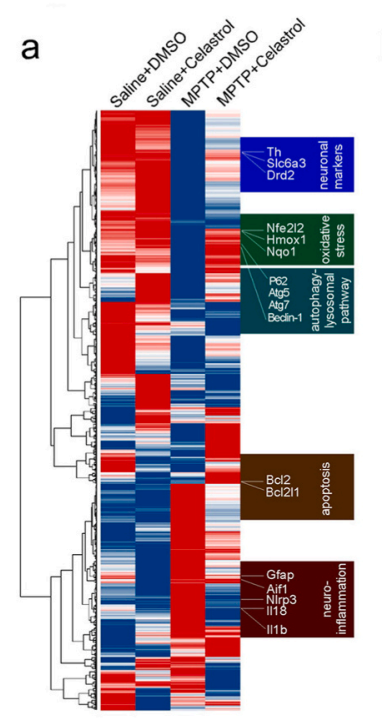

d

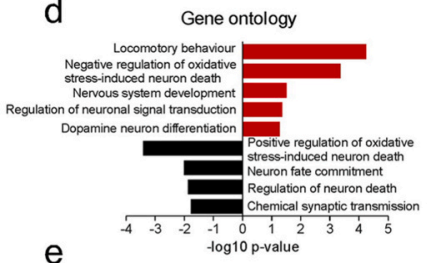

MPTP+Celastrol VS MPTP+DMSO

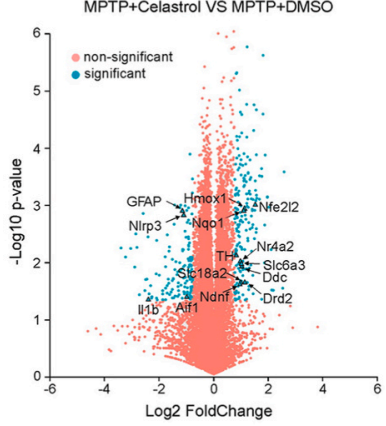

h

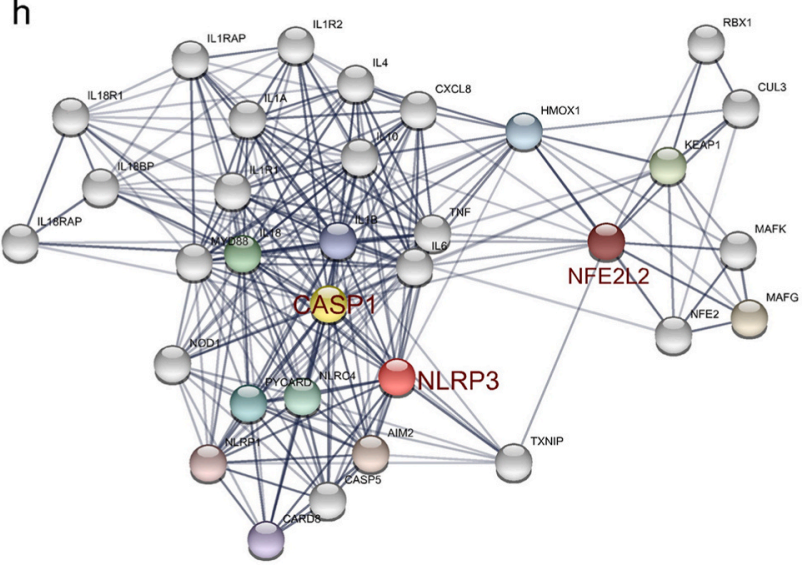

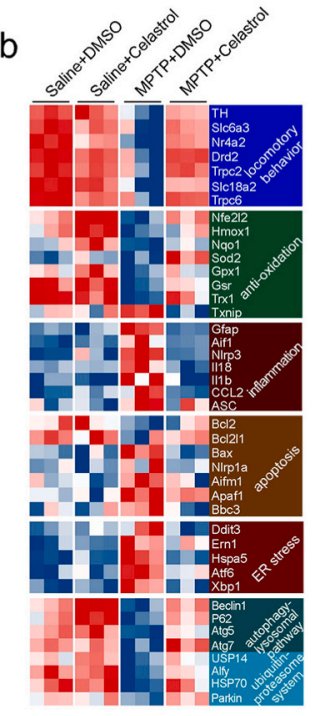

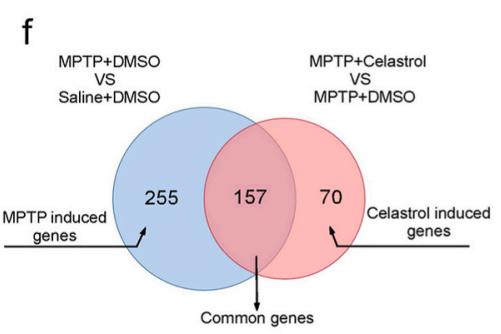

\begin{tabular}{|c|c|c|c|c|}
\hline \multicolumn{3}{|c|}{$\begin{array}{l}\text { MPTP+DMSO } \\
\text { VS } \\
\text { Saline+DMSO }\end{array}$} & \multicolumn{2}{|c|}{$\begin{array}{l}\text { MPTP+Celastrol } \\
\text { VS } \\
\text { MPTP+DMSO }\end{array}$} \\
\hline Gene Name & Log2 FoldChange & $p$-value & Log2 FoldChange & $p$ p-value \\
\hline $\mathrm{TH}$ & -1.007 & 0.011 & 0.788 & 0.012 \\
\hline Slc6a3 & -1.052 & 0.022 & 0.862 & 0.008 \\
\hline Drd2 & -1.334 & 0.035 & 1.231 & 0.038 \\
\hline Ne212 & -0.635 & 0.014 & 1.513 & 0.001 \\
\hline Hmox1 & -0.642 & 0.024 & 1.065 & 0.002 \\
\hline Nao1 & -0.521 & 0.064 & 1.014 & 0.002 \\
\hline GFAP & 1.226 & 0.013 & -1.435 & 0.008 \\
\hline Aif1 & 1.987 & 0.019 & -1.356 & 0.048 \\
\hline Nirp3 & 2.012 & 0.001 & -1.454 & 0.004 \\
\hline $111 \mathrm{~b}$ & 3.233 & 0.044 & -2.384 & 0.043 \\
\hline$A S C$ & 1.359 & 0.008 & . 0.781 & 0.048 \\
\hline
\end{tabular}

i
C Genes induced by celastrol
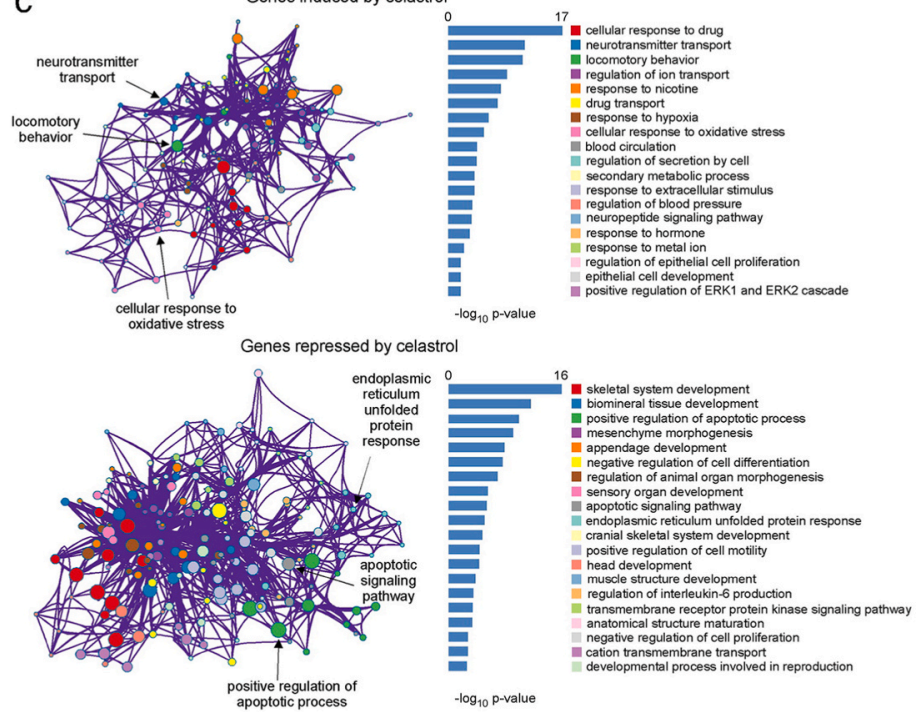

$-\log _{10}$ p-value
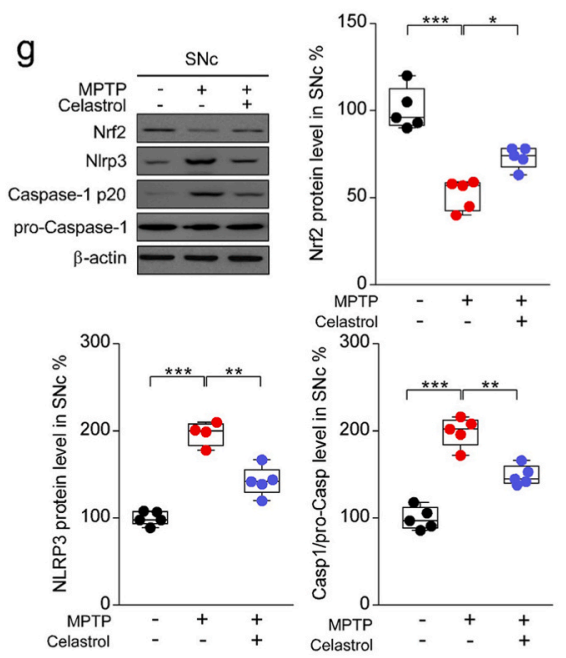

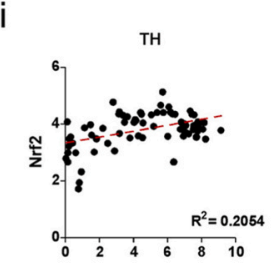

TH

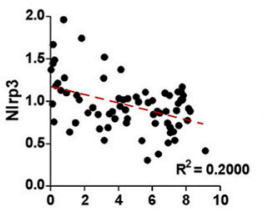

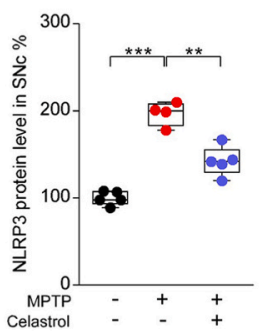

$\mathrm{TH}$

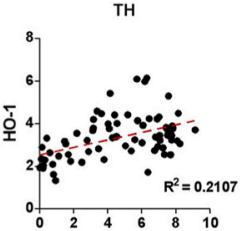

$\mathrm{TH}$

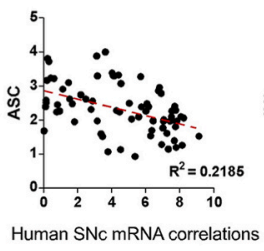

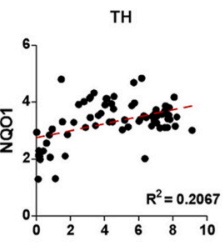

$\mathrm{TH}$

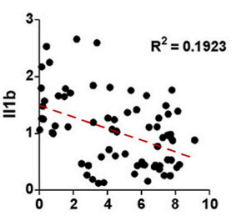

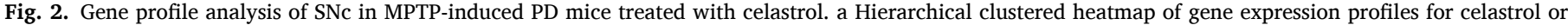

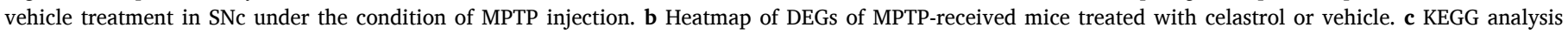

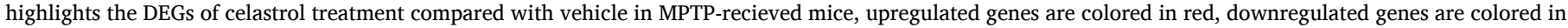

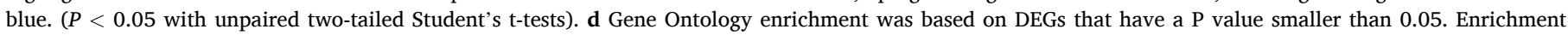

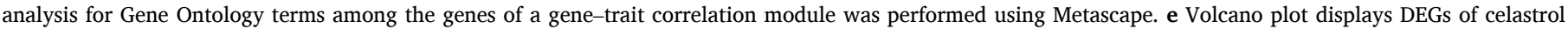

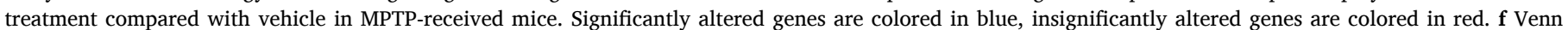

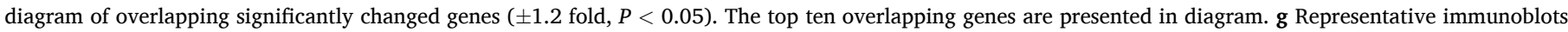

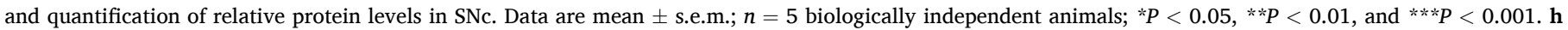

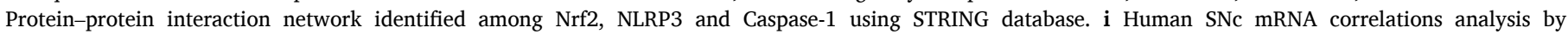
Gene network. 
a

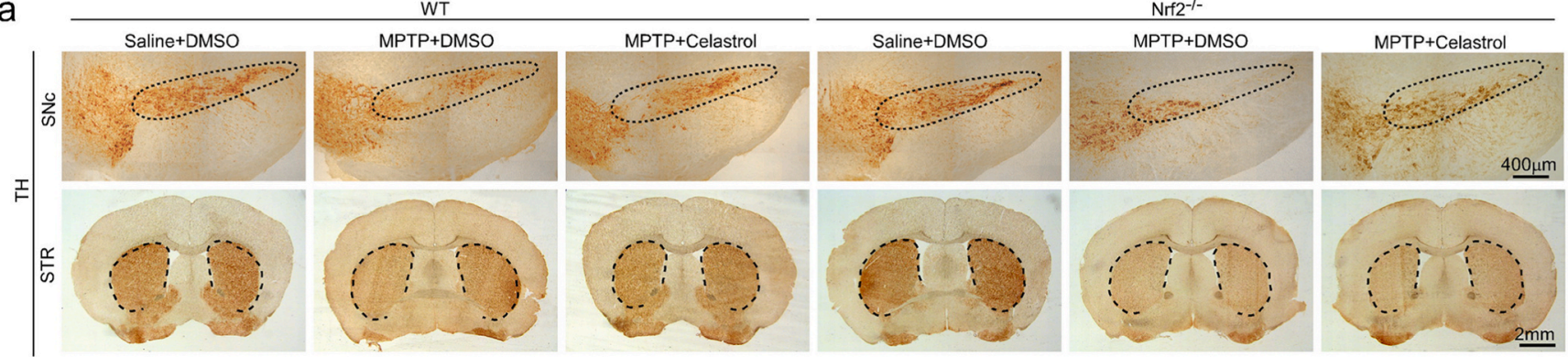

b
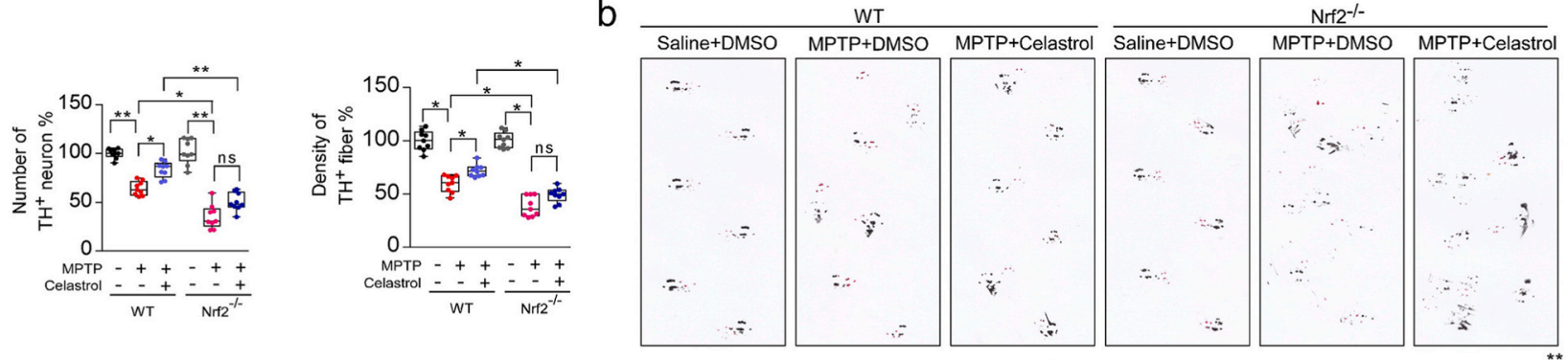

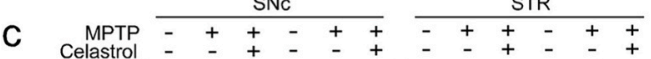
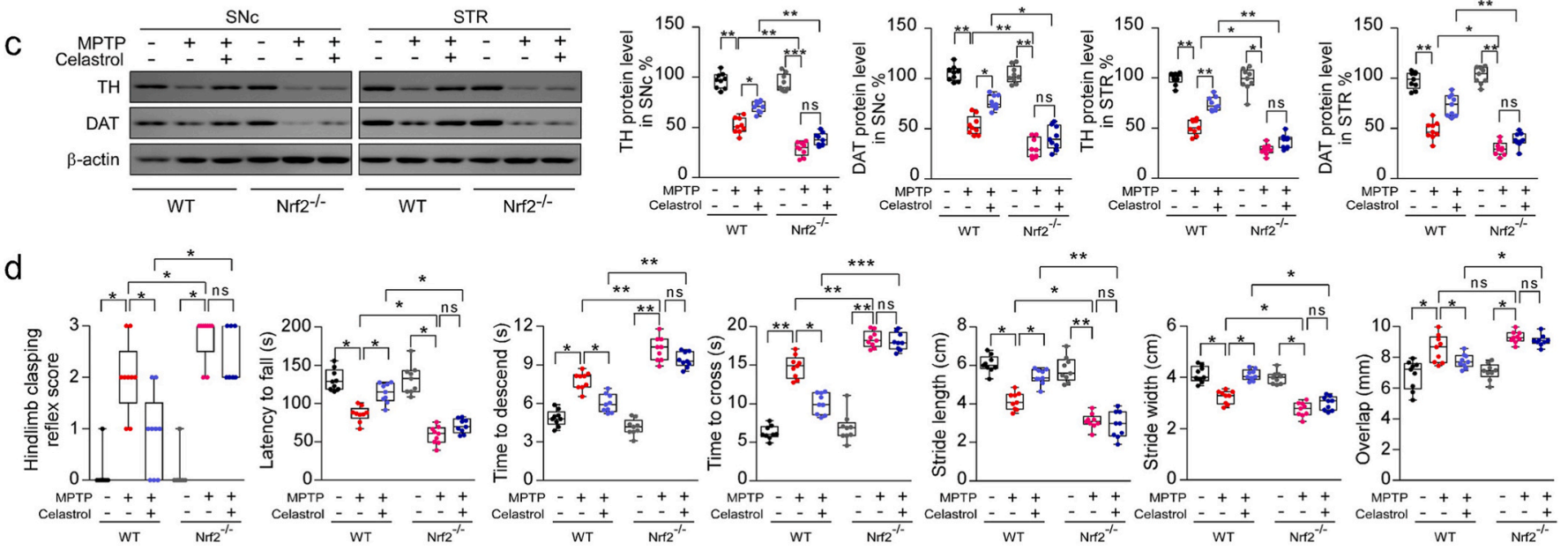

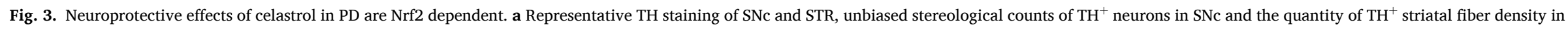

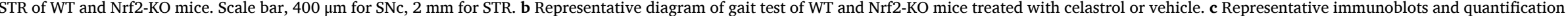

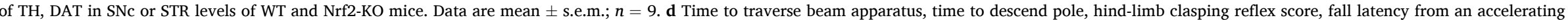
rotarod. Data are mean \pm s.e.m.; $n=9$. Two-way ANOVA followed by Tukey's post hoc test. ${ }^{*} P<0.05$, ${ }^{* *} P<0.01$, and ${ }^{* * *} P<0.001$. ns, not significant. 
b). Celastrol led to decreased expression of the genes associated with neuroinflammation (Gfap, Aif1, Nlrp3, IL18, ASC); apoptosis (Bcl2, Bax, Nlrp1a) and ER stress (Ddit3, Ern1, Hspa5, Xbp1) (Fig. 2a and b). To obtain deeper insights into signaling pathways regulated by celastrol, GO (gene ontogeny) and KEGG (Kyoto Encyclopedia of Genes and Genomes) analyses of DEGs were performed. We observed that celastrol led to a marked increase in the pathways related to locomotory behavior, dopamine neuron differentiation, neuronal signal transduction and neurotransmitter transport; whereas celastrol inhibited the pathways associated with inflammation, apoptotic process and neuron death (Fig. 2c and d). The volcano plot and Venn diagram showed that celastrol treatment led to an induction of TH, SLC6A3, Nfe2l2, Homx1 and NQO1, and a suppression of GFAP, AIF1, NLRP3, ASC, Caspase-1 and ASC under the condition of MPTP administration (Fig. 2e and f). Nrf2 (NFE2L2) is a transcription factor in anti-oxidative and antineuroinflammatory process in PD progression [27,42]. It's recently reported that the activation of NLRP3 inflammasome is a critical process associated with the PD pathogenesis, which triggers caspase- 1 activation and caspase1-mediated release of IL1 $\beta$ and IL18, and thus finally drives progressive dopaminergic neuropathology [2,32,35]. Celastrol caused an induction of Nrf2, and a decrease of NLRP3 and caspase-1 (Fig. 2g).By using STRING database, the Nrf2, NLRP3 and caspase-1 proteins and their functional interactions which formed the backbone of the cellular machinery were exhibited (Fig. 2h). Gene network analysis showed that the Nrf2, HO1, NQO1 mRNA levels were positively correlated with TH (tyrosine hydroxylase) mRNA levels, while NLRP3, ASC and IL1 $\beta$ mRNA levels were negatively correlated with mRNA levels of $T H$ in the human SNc, suggesting that Nrf2, HO1, NQO1 may underlie the neuroprotective effects of celastrol in PD; and NLRP3, ASC and IL1 $\beta$ may contribute to PD pathogenesis in humans (Fig. 2i). Together, these results suggest that Nrf2, NLRP3 and caspase-1 may be critical factors that underlie the pleiotropic effects of celastrol in PD treatment; and celastrol may protect against PD by activating Nrf2 and inhibiting NLRP3 inflammasome.

\subsection{The neuroprotective effects of celastrol against PD are Nrf2- dependent}

We have found that celastrol treatment led to a significant increase in Nrf2 levels under PD condition (Fig. 2). Thus, to further determine whether Nrf2 mediates the neuroprotective effects of celastrol, Nrf2-KO mice were employed. As previously reported, deficiency of Nrf2 caused a more profound loss of dopaminergic neurons in the SNc (Fig. 3a) [43], and celastrol failed to restore the loss of dopaminergic neurons and the decreased $\mathrm{TH}^{+}$fibers density in the Nrf2-KO mice (Fig. 3a). Likewise, the neuroprotective effect of celastrol on the TH and DAT (dopamine transporter) protein levels was impeded in Nrf2-KO mice (Fig. 3c). In addition, celastrol treatment failed to restore the impaired motor coordination and balance induced by MPTP in Nrf2-KO mice (Fig. 4b, d). These results suggest that Nrf2 may mediate the beneficial effects of celastrol against PD. Further, we investigated whether Nrf2 could be linked to the anti-neuroinflammatory action of celastrol in PD. We observed that mitigative effects of celastrol on the activation of $\mathrm{GFAP}^{+}$ astrocytes and $\mathrm{Iba}^{+}$microglia were halted in the SNc of MPTP administrated Nrf2-KO mice (Supplementary Fig. 10). Together, these observations suggest that celastrol may exert its anti-neuroinflammatory effects through activation of Nrf2, which may contribute to the neuroprotective role of celastrol in PD.

It is well established that activation of Nrf2 can exhibit the beneficial effects on neurons, astrocytes, and microglia in PD [24,44,45]. Thus, to better understand the mechanism underlying the neuroprotective properties of celastrol, we next asked in which cell types Nrf2 participates in the actions of celastrol. By using double staining of Nrf2 with cell-type specific markers (TH for dopaminergic neurons, GFAP for astrocytes and Iba1 for microglia) within SNc, we found that celastrol normalized Nrf2 level predominantly in dopaminergic neurons under the condition of MPTP, while celastrol had little effect on Nrf2 level in both astrocytes and microglia (Supplementary Fig. 11). Taken together, these findings suggest that Nrf2 may mediate the neuroprotective and anti-neuroinflammatory actions of celastrol mainly in dopaminergic neurons.

\subsection{Celastrol inhibits NLRP3 inflammasome to protect against PD}

The NLRP3 inflammasome has been observed in the SNc of both PD patients and PD animal models [2]. As previously reported [37,46], elevated levels of NLRP3 and ASC triggered by MPTP injection were also observed by western and immunohistochemistry analysis in this study (Supplementary Fig. 7a and b). Therefore, to determine whether NLRP3 is involved in the effects of celastrol in PD, the NLRP3-KO mice were employed. We found that the loss of dopaminergic neurons in the SNc and the decrease in density of $\mathrm{TH}^{+}$fibers in the STR (Fig. 4a) were relieved in MPTP-treated NLRP3-KO mice. The reduction in TH and DAT protein levels in the both SNc and STR (Fig. 4c) and the impaired motor function (Fig. 4b, d) were also mitigated in MPTP-treated NLRP3-KO mice. Notably, the neuroprotective effects of celastrol were unaltered in MPTP-treated NLRP3-KO mice (Fig. 4a-d), reflecting that NLRP3 may underlie the neuroprotective action of celastrol in PD, and celastrol may inhibit NLRP3 inflammasome to protect against PD. In addition, the activation of astrocytes and microglia in the both SNc and STR induced by MPTP was ameliorated in NLRP3-KO mice, and the anti-neuroinflammatory effects of celastrol were unaltered in MPTP-treated NLRP3-KO mice ()Supplementary Fig. 12. Together, these observations indicate that celastrol may suppress NLRP3 inflammasome to protect against PD.

\subsection{The effects of celastrol are mediated by Nrf2-NLRP3-caspase-1 axis}

It is reported that the caspase- 1 is activated in dopaminergic neurons in the SNc of PD models [2,32,35-37]. The deficiency of caspase-1 alleviates the loss of dopaminergic neurons in MPTP-induced PD mouse model [47]. The caspase-1 is the main effector of NLRP3 inflammasome [2]. Therefore, to determine whether caspase-1 underpins the neuroprotective action of celastrol in $\mathrm{PD}$, the caspase-1-KO mice were employed. We found that the caspase-1 deficiency profoundly restored the loss of dopaminergic neurons in the SNc (Fig. 5a), the reduced $\mathrm{TH}^{+}$ fibers in the STR (Fig. 5a), the decreased TH and DAT levels in the both SNc and STR (Fig. 5c), and the impaired motor function (Fig. 5b, d); while the neuroprotective actions of celastrol were unaltered (Fig. 5a-d) in caspase-1-KO mice, compared with the controls. These findings demonstrate that the neuroprotective effects of celastrol in PD may be mediated by inhibiting caspase- 1 , which also confirm that caspase- 1 may be a potential therapeutic target of PD. Since caspase-1 is associated with neuroinflammation during PD progression [48], we further decided to ascertain the role of caspase- 1 in the anti-neuroinflammatory action of celastrol. As shown in Supplementary Fig. 13, the increased number of $\mathrm{GFAP}^{+}$and $\mathrm{Iba}^{+}$cells in both SNc and STR was reduced by celastrol. Notably, the mitigative effect of celastrol on activation of astrocytes and microglia was parallel with that of the caspase-1-KO mice. These observations suggest that the inhibition of caspase- 1 may contribute to the anti-neuroinflammatory effects of celastrol. Accumulating evidence demonstrates that the activation of NLRP3 inflammasome has been found in both microglia and dopaminergic neurons within SNc of PD patients $[2,35,37,47,49]$. Thus, to investigate in which cell types the suppression of NLRP3 inflammasome may be involved in the actions of celastrol, the co-labelling analysis of ASC with TH, GFAP and Iba1 was performed. We observed that celastrol treatment led to a profound decrease in ASC levels in dopaminergic neurons, microglia and astrocytes treated with MPTP (Supplementary Fig. 14). These results suggest that celastrol may act on dopaminergic neurons, astrocytes and microglia to depress the activation of NLRP3 induced by MPTP.

We observed that celastrol treatment had little effects on the NLRP3, ASC, caspase-1, and IL1 $\beta$ in MPTP-treated Nrf2-KO mice 
a
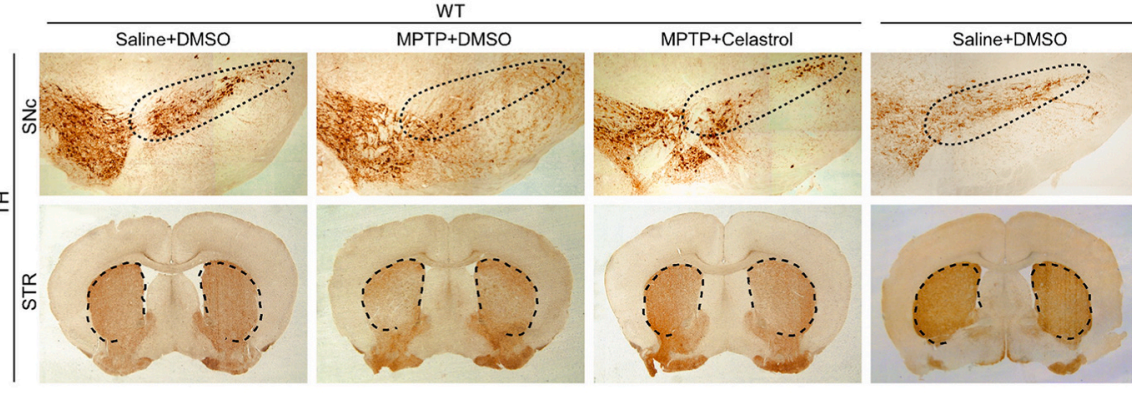

MPTP+DMSO

MPTP+Celastrol

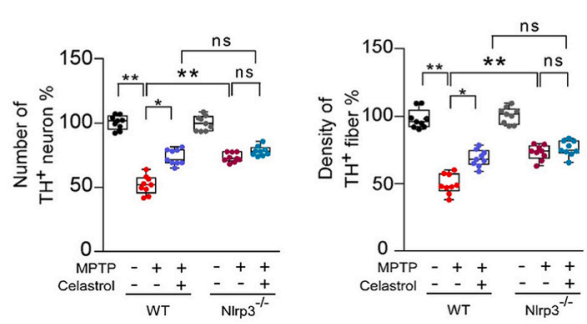

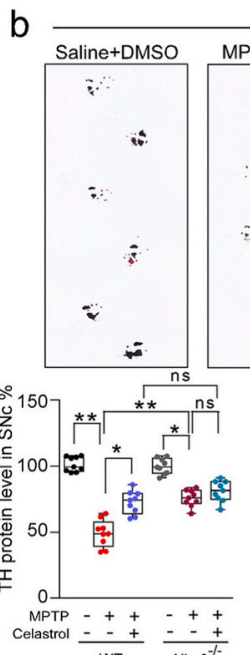
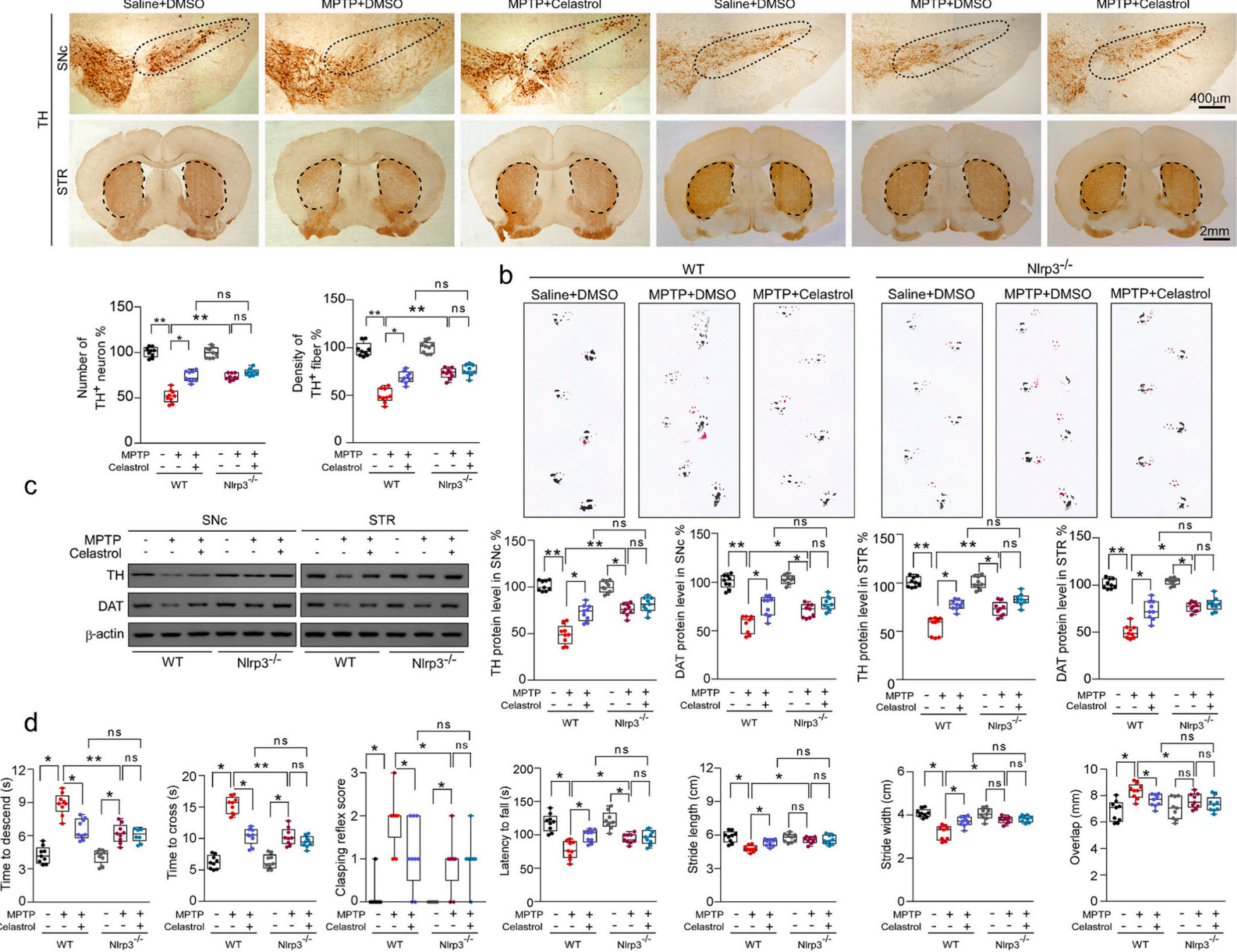

NIrp3 $3^{-1-}$
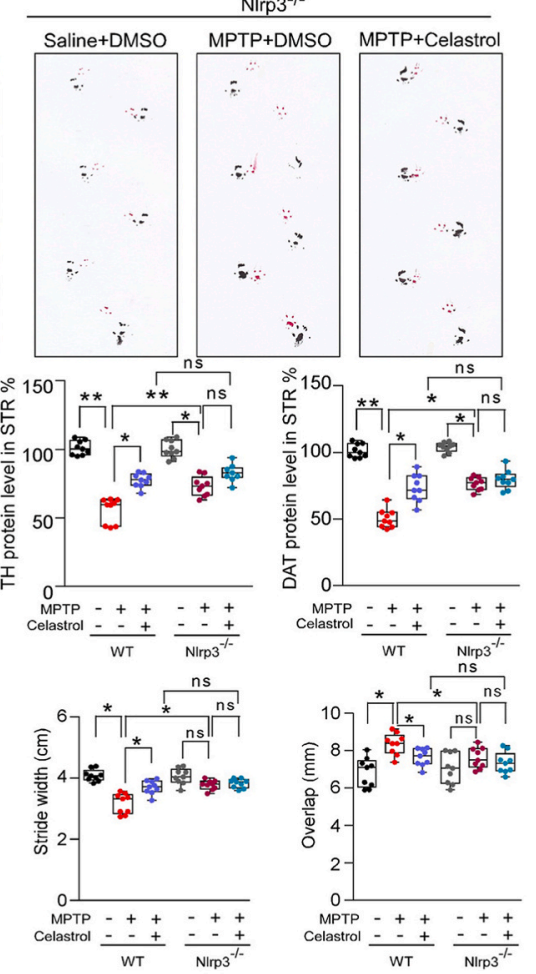

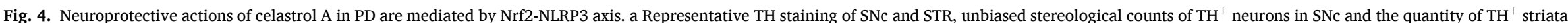

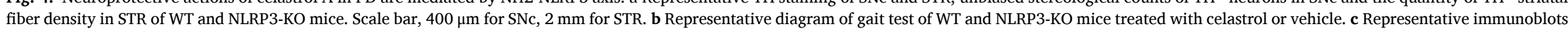

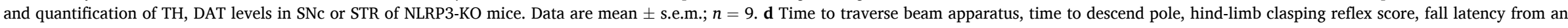
accelerating rotarod. Data are mean \pm s.e.m.; $n=9$. Two-way ANOVA followed by Tukey's post hoc test. ${ }^{*} P<0.05$, ${ }^{* *} P<0.01$, and ${ }^{* * *} P<0.001$. ns, not significant. 
a
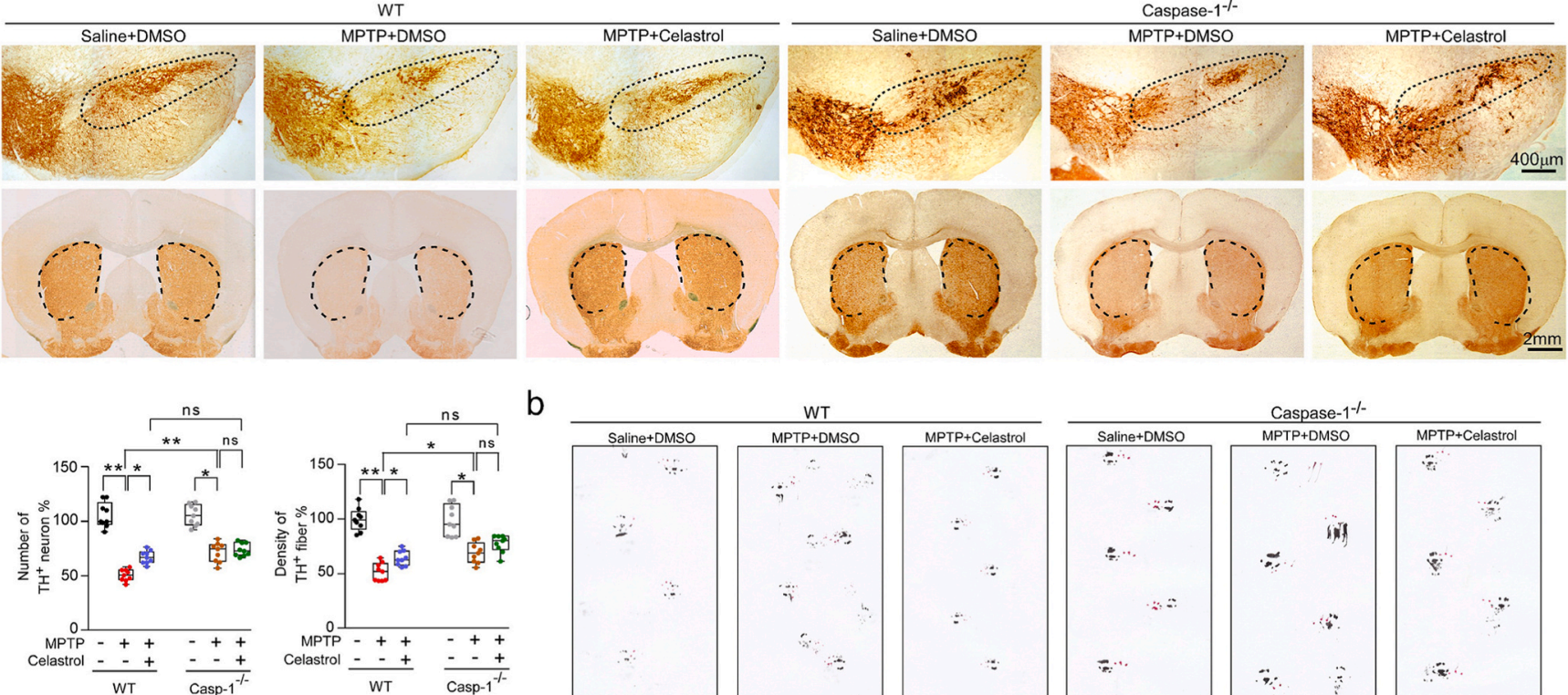

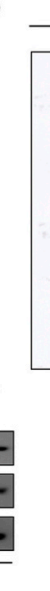
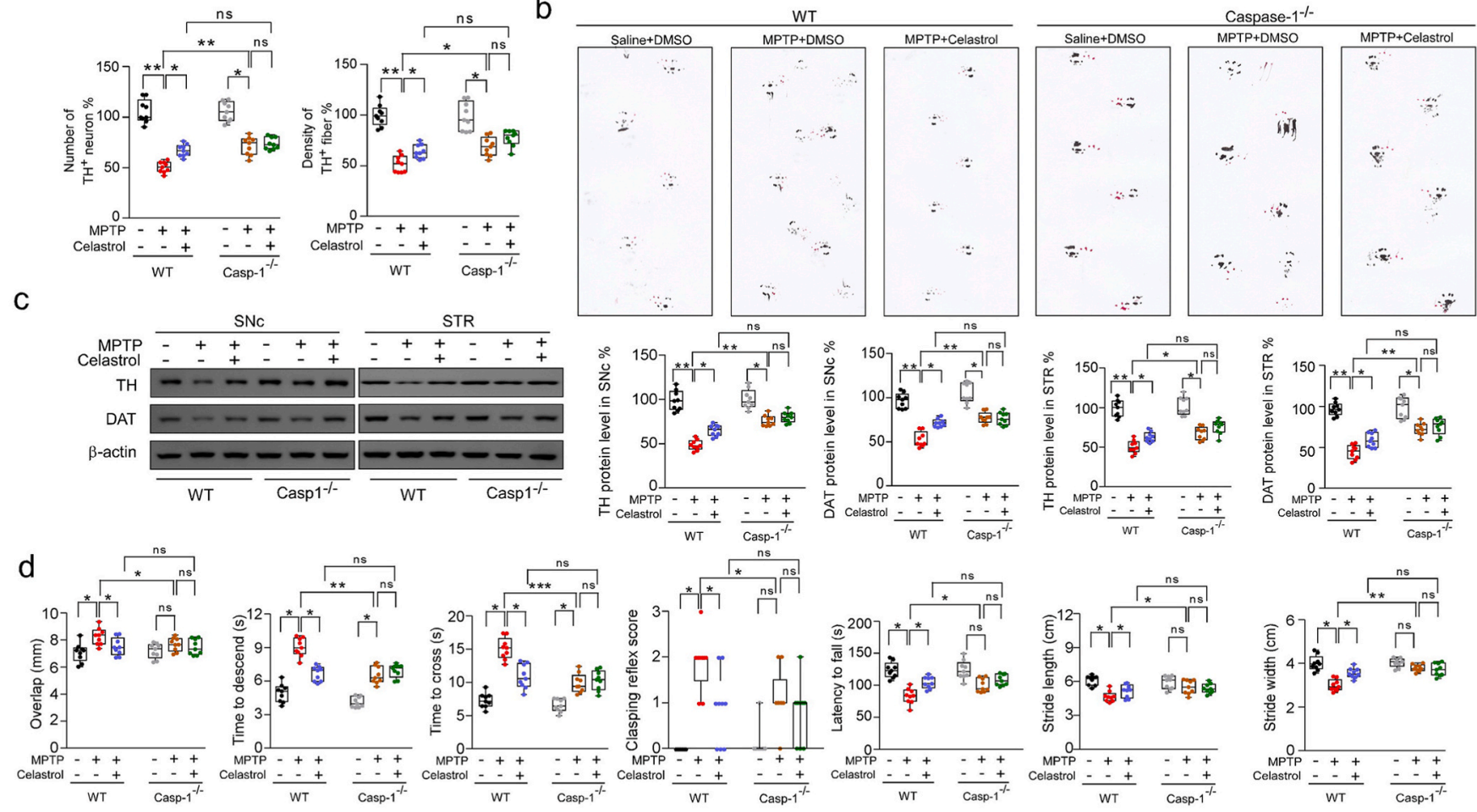

$\frac{\widehat{\sigma}^{200}}{\overline{\frac{0}{\omega}} 150}$
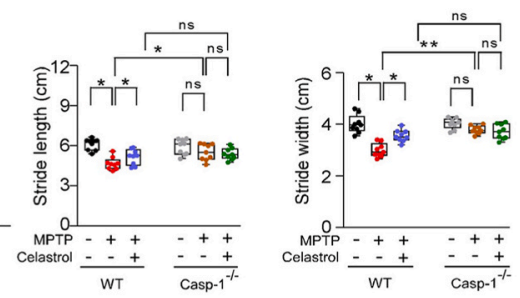

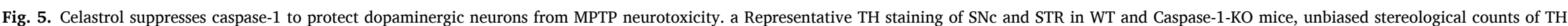

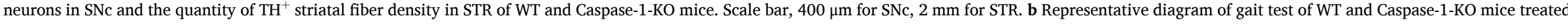

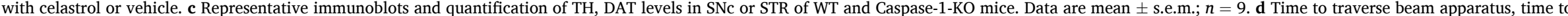

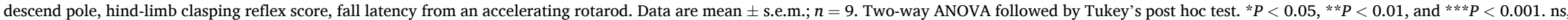
not significant. 
(Supplementary Fig. 15a), suggesting that the anti-PD effects of celastrol may be achieved by inhibiting NLRP3 inflammasome via activation of Nrf2. It is reported that pharmacological activator of Nrf2 protects against the MPTP-induced PD like symptom by inhibiting NLRP3 inflammasome [38]. Therefore, to ask whether the inhibition of NLRP3 by celastrol is dependent on the activation of Nrf2, western analysis was performed. We found that celastrol treatment caused increased protein levels of Nrf2 and its downstream factors (HO1 and NQO1) in NLRP3-KO mice (Supplementary Fig. 15b) suggesting that Nrf2 may be the upstream regulator of NLRP3 in the action of celastrol in PD. These results validate that celastrol may inhibit NLRP3 inflammasome to protect against PD via activation of Nrf2, suggesting that Nrf2-NLRP3 axis may underlie the favorable effects of celastrol in PD treatment. It is well documented that NLRP3 inflammasome is mainly composed of NLRP3, adaptor ASC and effector molecule caspase-1, which can promote IL1 $\beta$ and IL18 to exacerbate neuroinflammation during PD progression [35, 47]. We further observed that celastrol treatment elevated Nrf2, HO-1 and NQO1 in caspase-1-KO mice (Supplementary Fig. 15c), raising the possibility that activation of Nrf2 may inhibit caspase-1 to achieve the neuroprotective roles of celastrol in PD. Taken together, these findings suggest that the anti-PD effects of celastrol may be mediated by Nrf2-NLRP3-caspase-1 axis.

\subsection{Celastrol protects against neurodegeneration of dopaminergic neurons in SNc overexpressing human $\alpha-s y n$}

As the aggregation of $\alpha$-syn is the hallmark of PD, to model the neurodegeneration of the dopaminergic neurons, AAV-mediated human wild-type $\alpha$-syn (h- $\alpha$-syn) overexpression within SNc by stereotactic injection was performed, as shown in Fig. 6a. Co-labelling with TH (red) and $\mathrm{h}$ - $\alpha$-syn (green) ascertained that $\mathrm{h}-\alpha$-syn predominantly expressed within $\mathrm{SNc}$ at 21 days post injection (Fig. $6 \mathrm{~b}$ ). Similar results were observed that $\mathrm{h}$ - $\alpha$-syn overexpression caused marked loss of dopaminergic neurons in the SNc (Fig. 6c) [50], reduction of TH and DAT protein levels in both SNc (Fig. 6d) and STR (Supplementary Fig. 16c), and these changes were effectively reversed by celastrol. These results suggest that celastrol may protect against the neurodegeneration of the dopaminergic neurons induced by $\mathrm{h}-\alpha$-syn overexpression. Consistently, behavioral tests showed that the impaired motor function was also improved by celastrol (Fig. 6e and $\mathrm{f}$ ). In addition, $\mathrm{h}$ - $\alpha$-syn overexpression led to an increased number of $\mathrm{GFAP}^{+}$and Iba1 ${ }^{+}$cells, and elevated protein levels of GFAP and Iba1 in both SNc and STR (Supplementary Fig. 16a and b), which were also relieved by celastrol (Supplementary Fig. 16a and b). These results suggest that celastrol can mitigate the degeneration of the dopaminergic neurons and alleviate neuroinflammation induced by AAV-mediated h- $\alpha$-syn overexpression, highlighting the multiple neuroprotective properties of celastrol. Given that celastrol treatment led to restoration of the genes linked to ALP (alkaline phosphatase) and UPS (ubiquitin proteasome system) function in MPTP-induced PD mice, it is possible that celastrol may alleviate $\alpha$-syn pathology to impede the dopaminergic neuronal degeneration by enhancing ALP and UPS function.

As the diagram illustrates in Fig. $6 \mathrm{~g}$, our findings suggest that celastrol protects against the neurodegeneration of dopaminergic neurons and relieves the reactive astrocytes and microglia mediated neuroinflammation under the conditions of MPTP and h- $\alpha$-syn overexpression. The neuroprotective effects of celastrol in PD may be mainly mediated by Nrf2-NLRP3-caspase-1 axis. Collectively, we demonstrate favorably neuroprotective effects of celastrol which render it as a promising disease-modifying agent in the treatment of PD.

\section{Discussion}

The major findings of this paper are the observation that celastrol protects against the loss of dopaminergic neurons, maintains the nigrostriatal function, relieves the neuroinflammation, and mitigates motor deficit in both MPTP and human $\alpha$-synuclein overexpression-induced PD mouse models. These findings indicate that the multiple pharmacological properties of celastrol may contribute to its anti-PD effects, rendering celastrol as a promising candidate in the development of PD therapy.

The whole-genome deep sequencing analysis provides an ability to decipher the mechanism of PD pathogenesis [51,52]. In the present study, by using this approach, we identified a series of DEGs including Nrf2, NLRP3 and caspase-1 in SNc of MPTP-induced PD mice treated with celastrol. To obtain deeper insights into signaling pathways regulated by celastrol in $\mathrm{PD}$, we performed bioinformatic analysis, and we found that celastrol caused a marked increase in the pathways related to locomotory behavior, dopamine neuron differentiation, neuronal signal transduction, neurotransmitter transport and mitochondrial function, whereas celastrol inhibited the pathways associated inflammation, oxidative stress, apoptotic process, ER stress and neuron death. These findings highlight that celastrol may exert pleiotropic effects to protect against PD. Moreover, our findings reveal that Nrf2, NLRP3 and caspase-1 may critically underlie the pleiotropic effects of celastrol in PD treatment. Celastrol may protect against PD by activating Nrf2 and inhibiting NLRP3/caspase-1 pathway.

It is well documented that Nrf2 expresses in neurons, astrocytes and microglia [53-55] and plays an important role in PD pathogenesis [23]. Nrf2 overexpression protects against motor pathology and $\alpha$-synuclein aggregation [30]. Nrf2 deficiency exacerbates PD phenotypes in mice [24, 27]. Selegiline, which is currently used in PD treatment, displays anti-oxidative effect via Nrf2 [29]. Human studies demonstrate that a functional haplotype in the Nrf2 gene promoter confers higher transcriptional activity of Nrf2 and decreased incidence of PD in Swedish and Polish $[27,28]$. In this study, we observed celastrol activates Nrf2 in dopaminergic neurons. We found a profound loss of dopaminergic neurons and impaired motor function in Nrf2-KO mice under the condition of MPTP, which is consistent with a previous report [43]. Moreover, celastrol did not restore the loss of dopaminergic neurons, impaired motor function and neuroinflammation in Nrf2-KO mice. These findings reveal that Nrf2 may mediate the neuroprotective effects of celastrol in PD.

The neuroinflammation and oxidative stress play critical roles in PD pathogenesis [3,5,6,56-58]. Newly-discovered evidence suggests that NLRP3 inflammasome, which occurs in response to neurotoxicity (MPTP, rotenone), misfolded proteins ( $\alpha$-syn) and autophagy defect in the brain, has emerged as a critical neuroinflammatory mechanism that drives neurodegeneration, rendering it as an important therapeutic target in PD [2,32,35,49]. Accumulation of NLRP3 inflammasome can be observed in dopaminergic neurons of PD patients [32,36,47]. NLRP3 inflammasome is also highly expressed in the microglia of PD patients. A small-molecule NLRP3 inhibitor, MCC950, can inhibit NLRP3 inflammasome activation and effectively alleviate motor deficits, nigrostriatal dopaminergic degeneration, and accumulation of $\alpha$-synuclein aggregates [2]. Astragaloside IV ameliorates motor deficits and dopaminergic neuron degeneration via inhibiting NLRP3-mediated neuroinflammation and oxidative stress in PD mouse model [39]. Some studies have shown that dopamine released from dopaminergic neurons can inhibit NLRP3 activation in microglia through the DRD1-CAMP pathway $[33,59]$. Caspase-1 can be triggered within NLRP3 inflammasome, and is responsible for the production of inflammatory cytokines to promote the inflammatory response, which may exacerbate PD pathogenesis [47]. NLRP3-caspase-1 axis has emerged as promising therapeutic target of neurodegenerative diseases, such as PD and Alzheimer's disease $[60,61]$. These findings indicate that NLRP3 inflammasome is implicated in the PD pathogenesis by inducing the death of dopaminergic neurons $[2,32,35]$. In this study, by using NLRP3-KO and caspase-1-KO mice, we observed that deficiency of NLRP3 or caspase-1 exhibits profound mitigation of the loss of dopaminergic neurons, nigro-striatal function, neuroinflammation and motor function in MPTP-induced PD mice. Our findings suggest that celastrol may act on dopaminergic neurons and microglia to inhibit the activation of NLRP3 and caspase-1, which may underlie the neuroprotective effects of celastrol in PD. The NLRP3-caspase-1 axis may mediate the beneficial 
a
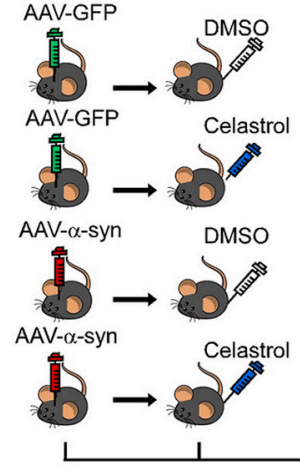

b

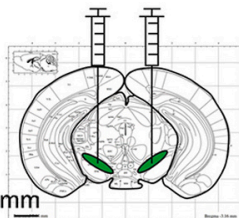

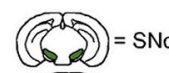

Bre

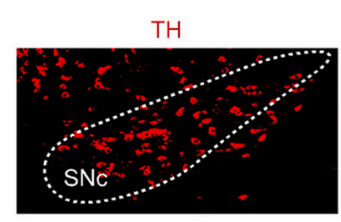

$C_{\text {AAV-GFP+DMSO }}$

AAV-GFP+Celastrol

(1)

and
Behavior Test

avior Test

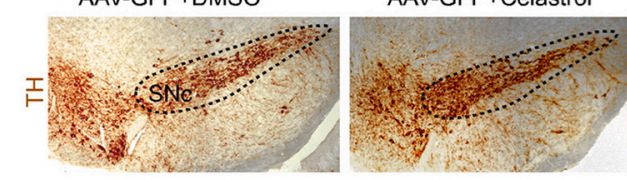

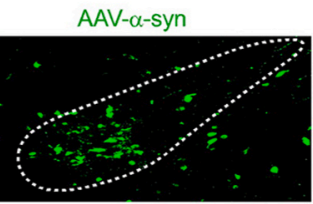

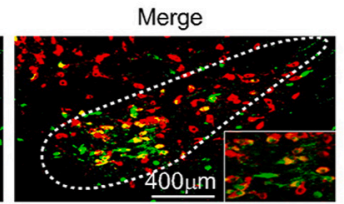

$\stackrel{\circ}{\circ} 150$ 吉 100 济異 단 $50-$ to

d

e $\vdash^{* * * * *}$
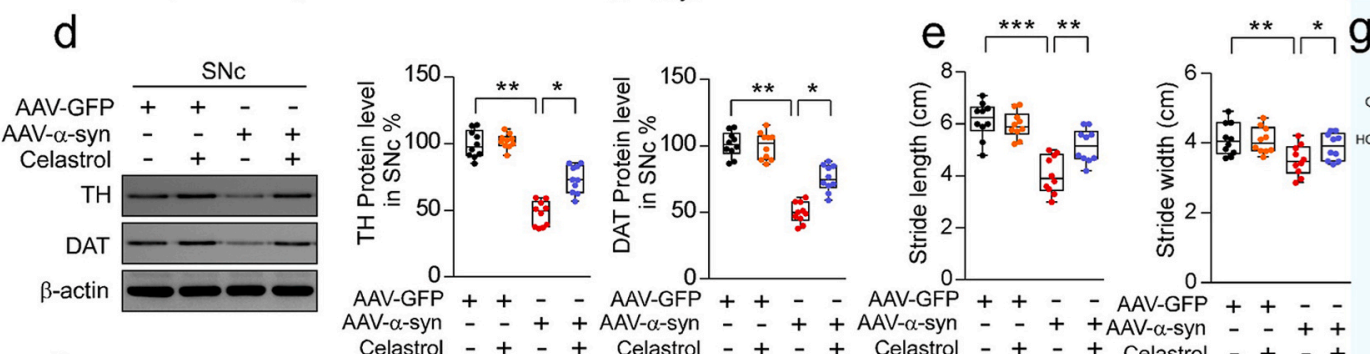

f Aav-asyn :

AAV- $\alpha$-syn
Celastrol - AAV-GFP + +- - AAV-GFP ++--
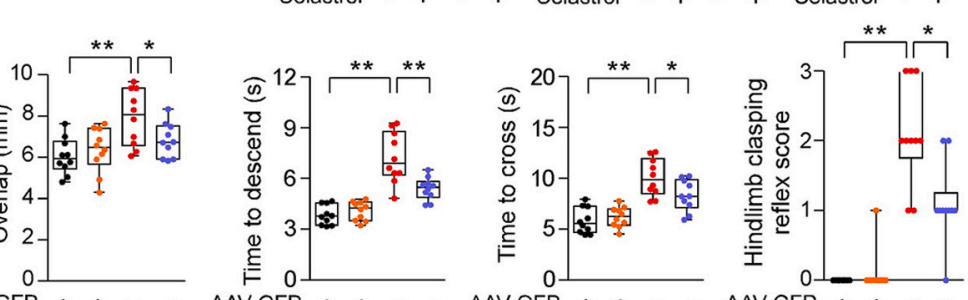

(200
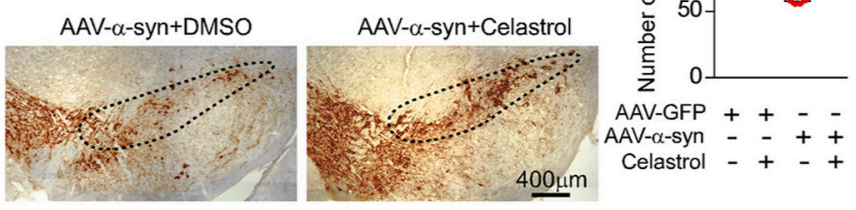

Celastrol --++

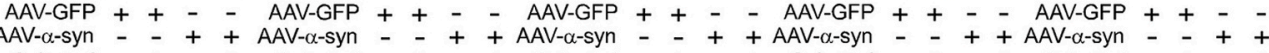

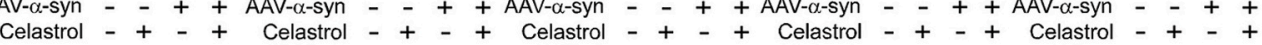

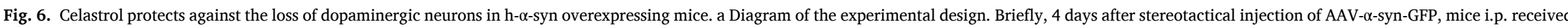

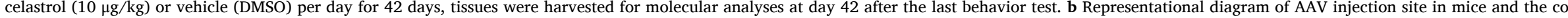

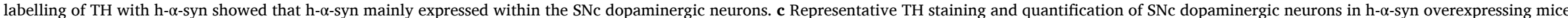

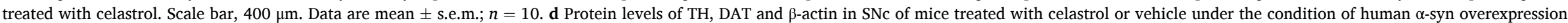

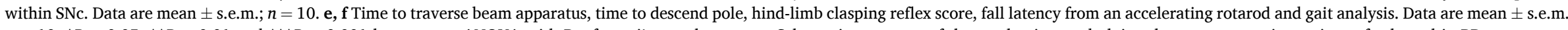
$n=10 .{ }^{*} P<0.05,{ }^{* *} P<0.01$ and ${ }^{* * *} P<0.001$ by one-way ANOVA with Bonferroni's post hoc test. $\mathrm{g}$ Schematic summary of the mechanism underlying the neuroprotective actions of celastrol in PD. 
effects of celastrol in PD.

It is reported that pharmacological activator of Nrf2 protects against the MPTP-induced PD like symptom by inhibiting NLRP3 inflammasome [38]. In this study, we found that celastrol had little effects on the NLRP3, ASC, caspase1, and IL1 $\beta$ in MPTP-treated Nrf2-KO mice; and Nrf2 may be the upstream regulator of NLRP3 in the actions of celastrol in PD. These results show that celastrol may inhibit NLRP3 inflammasome to protect against $\mathrm{PD}$ via Nrf2, indicating that Nrf2-NLRP3-caspase-1 axis may underlie the favorable effects of celastrol in PD treatment.

The hallmark of PD is the loss of dopaminergic neurons [6,9,57, 62-64]. PD is classified as a synucleinopathy, as $\alpha$-syn, is a major constituent of Lewy bodies and Lewy neurites within dopaminergic neurons $[63,65]$. There is growing evidence suggesting that $\alpha$-syn plays a central role in the etiology of PD [58], as it links familial and sporadic forms of PD. In this study, to model the neurodegeneration of PD and to ascertain the neuroprotective effects of celastrol in $\mathrm{PD}$, we performed the AAV vectors-mediated human wild-type $\alpha$-syn overexpression within the SNc, which is considered as a useful approach for preclinical validation of new therapeutic agents $[58,63,66]$. Our findings demonstrate that celastrol protects against the neurodegeneration of dopaminergic neurons and nigro-striatal system, and mitigates the impaired motor function in the mice overexpressing human $\alpha$-syn. As previously reported, celastrol protects human neuroblastoma SH-SY5Y cells from rotenone-induced injury through induction of autophagy [15]. We found that celastrol treatment led to a marked induction of the genes associated with ALP and UPS functions. These findings suggest that the promotion of ALP and UPS may be implicated in the neuroprotective effects of celastrol in PD.

It is reported that celastrol can cross the blood brain barrier to exert neuroprotective effects [67]. Nevertheless, the low water solubility, low oral bioavailability, short plasma half-life, narrow dosage-window and some side effects could hinder celastrol from clinical application as oral drugs [67-69]. To overcome these limitations, in our opinion, the chemical structure modification and new pharmaceutical dosage forms should be developed.

To date, there is no disease-modifying agents for the treatment of PD [9], and recent genetic gene therapies failed to show favorable anti-neurodegenerative effects $[10,11]$. Therefore, development of the neuroprotective agents to halt, delay or prevent the progression of PD remains a high priority. In this study, our findings suggest that celastrol protects against the loss of dopaminergic neurons, maintains nigro-striatal system function, mitigates neuroinflammation, and ameliorates motor defect via Nrf2-NLRP3-caspase-1 signaling pathway, suggesting broad neuroprotective properties of celastrol, which render it as a promising candidates of PD treatment, and also may help to improve the understanding of PD pathogenesis.

\section{Methods and materials}

Mice. C57BL/6 mice were purchased from Charles River Laboratories. Male mice were used for all the experiments at 8-10 weeks of age described in this study. Nrf2-KO knockout mice with a C57BL/6 background were kindly provided by Dr. Siwang Yu of Peking University [70]. NLRP3-KO mice (C57BL background) were kind gift from Dr. Han Xiao of Peking University Third Hospital [71]. Caspase1-KO mice were a kind gift from Dr. Changtao Jiang of Peking University Health Science Center [72]. We maintained mice on a $12 \mathrm{~h}$ light-dark cycle in a temperature-controlled high barrier facility with unrestricted access to food and water. During all procedures of experiments, the number of animals and their suffering by treatments were minimized.

\subsection{Reagents}

Celastrol were purchased from BOC Sciences (Shirley, NY), this compound was used as previously described [2]. Dimethyl sulfoxide
(DMSO) were obtained from Amresco (Solon, CA).

Administration of celastrol. Eight to ten weeks old male mice weighing 24-28 g were housed under standard conditions. After 1 week acclimatization, mice were injected with MPTP at a dose of $20 \mathrm{mg} / \mathrm{kg}$ i. p. for 5 days, as used previously [73], and the controls were administered an equal volume of saline $(0.9 \% \mathrm{NaCl})$. Then mice were administered celastrol (BOC Science Shirley, NY) at dose of 1, 10, 100, 1000 $\mu \mathrm{g} / \mathrm{kg}$ for 7, 14, 21 days. Celastrol was dissolved in DMSO $(25 \mu \mathrm{l})$ and administered to the mice intraperitoneally once a day, as previously reported [2]. All treatments were performed within $90 \mathrm{~min}$ before dark cycle and mice received celastrol or vehicle daily.

Celastrol treatment at the dosage levels of 10,100 and $1000 \mu \mathrm{g} / \mathrm{kg}$ relieved the reduction of tyrosine hydroxylase (TH) immunoreactivity in the SNc and STR (Supplementary Fig. 1b, Supplementary Fig. 2a), mitigated the impaired motor function (Supplementary Fig. 1d), attenuated the loss of dopaminergic neurons and led to a greater $\mathrm{TH}+$ fibers density in the STR (Supplementary Fig.3), as compared with controls. In addition, celastrol administration at the doses of $10 \mu \mathrm{g} / \mathrm{kg}$ in treatment courses for 7, 14, 21 days showed significant neuroprotective actions in MPTP-induced PD mouse model (Supplementary Fig. 4a,c, Supplementary Fig. 5a, Supplementary Fig. 6). Celastrol alone had no obvious effects on the TH immunoreactivity in the both SNc and STR and motor function (Supplementary Fig. 1c, e, Supplementary Fig. 2b, Supplementary Fig. 4b, d, and Supplementary Fig. 5b). Based on these observations, we finally decided to initiate treatment with celastrol $(10 \mu \mathrm{g} /$ $\mathrm{kg}$ ) for 7 days in MPTP-induced PD mouse model.

Table S1

Antibodies used in this study.

\begin{tabular}{|c|c|c|c|}
\hline Antibodies & Source/Cat. No. & Host & Dilution \\
\hline$\alpha$-synuclein & Abcam (ab138501) & Rabbit & $\begin{array}{l}1: 2,000 \\
(\mathrm{WB}) \\
1: 500(\mathrm{IHC}, \\
\mathrm{IF})\end{array}$ \\
\hline $\begin{array}{l}\text { p- } \alpha \text {-synuclein } \\
\text { Ser129 }\end{array}$ & Biolegend (825701) & Mouse & $\begin{array}{l}1: 500(\mathrm{IHC}, \\
\text { IF) } \\
1: 2000(\mathrm{WB})\end{array}$ \\
\hline $\begin{array}{l}\text { Tyrosine Hydroxylase } \\
\text { (TH) }\end{array}$ & $\begin{array}{l}\text { Millipore (AB152) } \\
\text { Santa Cruz (sc-25269) }\end{array}$ & $\begin{array}{l}\text { Rabbit } \\
\text { Mouse }\end{array}$ & $\begin{array}{l}1: 2,000 \\
(\mathrm{WB}) \\
1: 500(\mathrm{IHC}, \\
\mathrm{IF})\end{array}$ \\
\hline $\begin{array}{l}\text { Dopamine transporter } \\
\text { (DAT) }\end{array}$ & Bioss (bs-1714R) & Rabbit & $\begin{array}{l}1: 1,000 \\
\text { (WB) }\end{array}$ \\
\hline NLRP3 & $\begin{array}{l}\text { Cell signaling technology } \\
\text { (13158S) }\end{array}$ & Rabbit & $\begin{array}{l}1: 2,000 \\
\text { (WB) } \\
1: 500(\mathrm{IHC}, \\
\text { IF) }\end{array}$ \\
\hline Nrf2 & $\begin{array}{l}\text { Abcam (ab31163) } \\
\text { Santa Cruz (sc-365949) }\end{array}$ & $\begin{array}{l}\text { Rabbit } \\
\text { Mouse }\end{array}$ & $\begin{array}{l}1: 2000(\mathrm{WB}) \\
1: 500(\mathrm{IHC}, \\
\text { IF) }\end{array}$ \\
\hline P20 Caspase-1 & Bioss (bs-10442R) & Rabbit & $\begin{array}{l}1: 500(\mathrm{IHC}, \\
\mathrm{IF})\end{array}$ \\
\hline ASC & Bioss (bs-6741R) & Rabbit & $\begin{array}{l}1: 500(\mathrm{IHC}, \\
\mathrm{IF})\end{array}$ \\
\hline IL1 $\beta$ & Bioss (bs-6319R) & Rabbit & $1: 2000(\mathrm{WB})$ \\
\hline GFAP & $\begin{array}{l}\text { Bioss (bs-0199R) } \\
\text { Santa Cruz (sc-33673) }\end{array}$ & $\begin{array}{l}\text { Rabbit } \\
\text { Mouse }\end{array}$ & $\begin{array}{l}1: 500(\mathrm{WB}) \\
1: 200(\mathrm{IHC}, \\
\mathrm{IF})\end{array}$ \\
\hline Iba1 & $\begin{array}{l}\text { Wako (019-19741) } \\
\text { GeneTex (GTX632426-S) }\end{array}$ & $\begin{array}{l}\text { Rabbit } \\
\text { Mouse }\end{array}$ & $\begin{array}{l}1: 500(\mathrm{IHC}) \\
1: 1,000 \\
(\mathrm{WB})\end{array}$ \\
\hline$\beta$-actin & Abcam (ab7817) & Mouse & $1: 2000(\mathrm{WB})$ \\
\hline
\end{tabular}

Immunohistochemistry and immunofluorescence. Immunohistochemistry and immunofluorescence were performed on $30-\mu \mathrm{m}$ thick serial brain sections. Primary antibodies and working dilutions are detailed in Table S1. Mice were perfused with PBS and 4\% PFA, and brains were removed, followed by fixation in $4 \%$ PFA overnight and transferred to $20 \%$ $30 \%$ gradient sucrose for cryoprotection, and subsequently freezed in OCT 
compound (Sakura FineTech, Tokyo). For histological studies, brain slices were blocked with $10 \%$ goat serum in PBS with $0.2 \%$ Triton X-100 and incubated with TH, GFAP or Iba1 antibodies. After washing with PBS three times, brain tissues were used appropriate biotin secondary antibody, followed by avidin-biotin complex and visualized with $3,3^{\prime}$-diaminobenzidine (DAB) peroxidase substrate, and photographed by light microscope (Leica DMI 4000B, Wetzlar, Germany). The number of TH-positive dopaminergic neurons, and the number of microglia and astrocytes in the SNc region, and the density of TH positive fibers in the STR were measured with ImageJ software. For immunofluorescent studies, dual-antigen immunofluorescence was performed to detect the expression level of Nrf2, NLRP3 and Caspase-1 in dopaminergic neurons, astrocytes or microglia (TH, GFAP and Iba1). Sections were washed in PBS and incubated with a mixture of Alexafluor 488- and 594- conjugated secondary antibodies (1:500) at room temperature for $120 \mathrm{~min}$. Sections were mounted with VectaShield medium (Vector Laboratories) and analyzed by detecting fluorescin using a fluorescence microscope (Leica DMI 4000B, Wetzlar, Germany). The selected area in the signal intensity range of the threshold was measured using ImageJ software. In this study, the statistics of IHC-related experimental results (such as $\mathrm{TH}+$ neurons) were counted by an investigator who was blind to genotypes or treatment groups by using image processing software. The thickness of each SNc and STR brain slice is $30 \mu \mathrm{m}$, and every 5 slices in the coronal direction are taken for IHC staining, then the staining result of the SNc area was obtained by using a fluorescence microscope (Leica DMI 4000B, Wetzlar, Germany). The total population estimate was calculated using optical fractionator estimates [74].

Total protein extraction and western analysis. SNc and STR were homogenized with a Polytron in ice-cold RIPA buffer [1\% Trinton X 100; 10 mM Na2HPO4 (Sodium phosphate); $150 \mathrm{mM} \mathrm{NaCl}$ (Sodium chloride); 1\% DOC (Sodium deoxycholate or deoxycholic acid); 5 mM EDTA; $5 \mathrm{mM} \mathrm{NaF}$ (sodium Fluoride); $0.1 \%$ SDS] supplemented with protease and phosphatase inhibitors (catalog \#P8340 and \#P2850; Sigma), sonicated and cleared by centrifugation $\left(10,000 \times g, 10 \mathrm{~min}\right.$, at $\left.4{ }^{\circ} \mathrm{C}\right)$. Western procedure was previously described [75]. Protein concentration in the supernatant was determined by BCA assay (Aidlab; PP01). Protein $(5 \mu \mathrm{g})$ in $1 \times$ sample buffer $[62.5$ $\mathrm{mM}$ Tris•Cl (pH 6.8), $2 \%$ (wt/vol) SDS, $5 \%$ glycerol, $0.05 \%$ (wt/vol) bromophenol blue] was denatured by boiling at $100^{\circ} \mathrm{C}$ for $5 \mathrm{~min}$ and separated on $8 \%$ sodium dodecyl sulfate poly acrylamide (SDS-PAGE) gels and transferred onto nitrocellulose membrane (Pall Corporation; T60327) by electrophoresis. Blots were blocked in 5\% nonfat milk in Tris-buffered saline and Tween 20 (TBST) for $2 \mathrm{~h}$ at room temperature and probed with primary antibody in $5 \%$ BSA-TSBT overnight at $4{ }^{\circ} \mathrm{C}$. After overnight incubation, the blots were washed three times in TBST for $15 \mathrm{~min}$, followed by incubation with HRP-conjugated secondary antibody in TBST with $5 \%$ nonfat milk for 2 $\mathrm{h}$ at room temperature. Following three cycles of $15 \mathrm{~min}$ washes with TBST, the blots were developed using an Enhanced Chemiluminescence assay (BIO-Rad). Densitometry analysis was performed on scanned Western blot images using the ImageJ software (NIH).

Behavioral tests. To evaluate the beneficial effect of celastrol on behavioral deficits in MPTP-induced PD mouse model and AAV vectorsmediated human $\alpha$-syn overexpression induced PD model, mice were assessed by beam traversal, pole test, rotarod test, hindlimb reflect scoring and gait test. Before pharmacological testing, mice were handled for a week by the same operator to reduce stress, and trained for behavioral tests as described below until their motor performance became reproducible. Motor function for all animals was tested between 10:00-16:00 in the lights-on cycle. All tests were performed similarly to previous studies [76-78]. Apparatus was cleaned with 75\% ethanol after each trial.

Beam traversal. A $1 \mathrm{~m}$ beam was constructed of four segments of $0.25 \mathrm{~m}$ in length. Each segment was of thinner widths $3.5 \mathrm{~cm}, 2.5 \mathrm{~cm}, 1.5 \mathrm{~cm}$, and 0.5 $\mathrm{cm}$, with $1 \mathrm{~cm}$ overhangs placed $1 \mathrm{~cm}$ below the surface of the beam. The widest segment acted as a loading platform for the animals and the narrowest end placed into home cage. Animals had two days of training to traverse the length of the beam before testing. On the first day of training, animals received 1 trial with the home cage positioned close to the loading platform and guided the animals forward along the narrowing beam. Animals received two more trials with limited or no assistance to encourage forward movement and stability on the beam. On the second day of training, animals had three trials to traverse the beam and generally did not require assistance in forward movement. On the third day, animals were timed over three trials to traverse from the loading platform and to the home cage. Timing began when the animals placed their forelimbs onto the $2.5 \mathrm{~cm}$ segment and ended when one forelimb reached the home cage.

\subsection{Pole test}

Mice were acclimatized in the behavioral procedure room for at least $30 \mathrm{~min}$. A $75 \mathrm{~cm}$ long pole, $1 \mathrm{~cm}$ in diameter, wrapped with nonadhesive shelf liner to facilitate the animals grip, was placed into the home cage. Animals received two days of training to descend from the top of the pole and into the home cage. On the test day, animals were placed head-down on the top of the pole and timed to descend back into the home cage. Timing began when the experimenter released the animal and ended when one hind-limb reached the home cage base.

Rotarod test. Rotarod was performed by placing the mice on an accelerating rod (4-40 r.p.m. over the course of a 5-min trial, 3 trials/ day, 20-min inter-trial interval, for three days). All animals were trained 3 days before rotarod test. Latency to fall were recorded. Motor test data were presented as the percentage of the mean duration (three trials) on the rotarod compared to the control.

Hindlimb reflect scoring. Animals were gently lifted upward by the mid-section of the tail and observed over 5-10 s. Animals were assigned a score of $0,1,2,3$ based on the extent to which the hindlimbs clasped inward. 0 , indicating no clasping, was given to animals that freely moved both their limbs and extended them outward. A score of 1 was assigned to animals which clasped one hindlimb inward for the duration of the restraint or if both legs exhibited partial inward clasping. A score of 2 was given if both legs clasped inward for the majority of the observation, but still exhibited some flexibility. A score of 3 was assigned if animals displayed complete paralysis of hindlimbs that immediately clasped inward and exhibited no signs of flexibility.

\subsection{Gait test}

The testing apparatus is made of a gray acrylic board ( $3 \mathrm{~mm}$ thick), and consists of a runway ( $10 \mathrm{~cm}$ width, $60 \mathrm{~cm}$ length, $12 \mathrm{~cm}$ height) with non-slippery white paper and a dark goal box $(16 \mathrm{~cm}$ width, $10 \mathrm{~cm}$ length, $12 \mathrm{~cm}$ height). On the first training day, mice were habituated to the apparatus for $2 \mathrm{~min}$, then their forepaws and hindpaws were painted red and black with non-toxic dyes and trained to run to the goal box (training trial). In test trials, mice were made to run the runway in the same manner (cut-off time $60 \mathrm{~s}$ maximum). The footprint patterns were analyzed for three parameters (stride length, stride width, and overlap), prints near the start and the goal being excluded because of the effects of acceleration or deceleration. Stride length was measured as the average distance between each forepaw and hindpaw footprint. Stride width was measured as the average distance between the right and left footprint of each forepaw and hindpaw. Overlap was measured as the average distance between the center of forepaw and hindpaw footprints on the same side. At least four values were measured in each trial for each parameter.

Quantitative real-time PCR. Total RNA was extracted from adipose tissues and hypothalamus using TRIzol reagent. Quantification and integrity analysis of total RNA was performed by running $1 \mu$ of each sample on NanoDrop 5500 (Thermo). The cDNA was prepared by reverse transcription (TransScript one-step gDNA removel and cDNA synthesis Super MiX). The relative expression of mRNAs was determined by the SYBR Green PCR system (Bio-Rad). The relative expression of genes of interest was calculated by comparative $\mathrm{Ct}$ method and GAPDH was used as an endogenous control. GAPDH RNA was chosen as the housekeeping gene. Sequences of the primers used for real-time qPCR are available in table S2: Primers used in the present study. 
Table S2

Primers used in this study.

\begin{tabular}{|c|c|c|}
\hline Primer & Forward Primer $5^{\prime}-3^{\prime}$ & Reverse Primer $5^{\prime}-3^{\prime}$ \\
\hline TNF-a & GACGTGGAACTGGCAGAAGAG & TTGGTGGTTTGTGAGTGTGAG \\
\hline IL-1beta & GAAATGCCACCTTTTGACAGTG & TGGATGCTCTCATCAGGACAG \\
\hline IL-6 & TAGTCCTCСТACСССАATT & TTGGTCCTTAGCCACTCCTT \\
\hline IL-8 & CTTACTGACTGGCATGAGGATCA & GCAGCTCTAGGAGCATGTGG \\
\hline NF-kB & AGAGGGGATTTCGATTCCGC & CCTGTGGGTAGGATTTCTTGTTC \\
\hline iNOS & AAGTCGGGGAGGAGTTTCACG & GGAGCTTAACGGAGGCTTGGA \\
\hline GFAP & CGGAGACGCATCACCTCTG & TGGAGGAGTCATTCGAGACAA \\
\hline Iba-1 & CTTGAAGCGAATGCTGGAGAA & GGCAGCTCGGAGATAGCTTT \\
\hline PGC1a & TCCACTGTCCACCTGATTGA & TGGCATGTAGTCTGGAGCTG \\
\hline UCP2 & ATATAAGCCCAACTGCAGGAAA & GTCAAAGTCAGTGCGTTCAAAG \\
\hline MFN1 & CCGGTCGATGCAACGAGTGATGAGG & GCCTCCAGCTTGCATGATCTCCGG \\
\hline MFN2 & TACGAAGTGGTCTGTGGGCAATCA & TCAGCTTGTTGGACAGGGCTATCA \\
\hline Drp1 & CCACAGGACAGTACAGGATG & TCAAGTCGTGCTGAATAATACC \\
\hline OPA1 & ATGGTGAAGCTGATCGAGAGC & GGCATA TTCAGTAATAGAGGC \\
\hline PINK1 & CGCCTATGAAATCTTTGGGC & GCACTGCCTTGGCCATAGAA \\
\hline Tfam & AAAAGTCGGGGTCTCTCTGAC & CAGTCGGTCCAAAATTCTTGTGA \\
\hline Nrf1 & GGACCCAGTCAGGTTGGTG & TCCTGGTCTAAGCCGATTATCAT \\
\hline Fis1 & ATGTGGCACTTTTATGTGACCA & CCCCAGGTTCTTGTCGTCTTG \\
\hline Bcl-2 & CCTGTGGATGACTGAGTACC & CCCACTCGTAGCCCCTCT \\
\hline Bcl-xl & TGCCCAAGCTCAACTTGAAGA & TGGCCGTAGACGGTTGTCATA \\
\hline Bax & GGCGAATTGGAGATGAAC & CCGAAGTAGGAGAGGAGG \\
\hline Caspase3 & CCCCTGTCATCTTTTGTCCCT & AGCTGGCAGAATAGCTTATTGAG \\
\hline Caspase9 & GGCTGTTAAACCCCTAGACCA & TGACGGGTCCAGCTTCACTA \\
\hline PUMA & CATGGGACTCCTCСССТTAC & CACCTAGTTGGGCTCCATTT \\
\hline CHOP & AAGCCTGGTATGAGGATCTGC & TTCCTGGGGATGAGATATAGGTG \\
\hline ATF6 & TCGCCTTTTAGTCCGGTTCTT & GGCTCCATAGGTCTGACTCC \\
\hline GRP78 & ACTTGGGGACCACCTATTCCT & GTTGCCCTGATCGTTGGCTA \\
\hline XBP1 & AGCTTTTACGGGAGAAAACTCAC & CCTCTGGAACCTCGTCAGGA \\
\hline ERN1 & ACACTGCCTGAGACCTTGTTG & GGAGCCCGTCCTCTTGCTA \\
\hline Nrf2 & TTCTTTCAGCAGCATCCTCTCCAC & ACAGCCTTCAATAGTCCCGTCCAG \\
\hline $\mathrm{HO}-1$ & CAAGCCGAGAATGCTGAGTTCATG & GCAAGGGATGATTTCCTGCCAG \\
\hline NQO1 & GCGAGAAGAGCCCTGATTGTACTG & TCTCAAACCAGCCTTTCAGAATGG \\
\hline GCLC & ACATCTACCACGCAGTCAAGGACC & CTCAAGAACATCGCCTCCATTCAG \\
\hline GCLM & GCCACCAGATTTGACTGCCTTTG & TGCTCTTCACGATGACCGAGTACC \\
\hline Gapdh & AGGTCGGTGTGAACGGATTTG & TGTAGACCATGTAGTTGAGGTCA \\
\hline
\end{tabular}

Whole-genome sequencing analysis. Total RNA was extracted from SNc using TRIzol reagent. Quantification and integrity analysis of total RNA was performed by running $1 \mu \mathrm{l}$ of each sample on NanoDrop 5500 (Thermo). A total amount of $3 \mu \mathrm{g}$ RNA per sample was used as input material for the RNA sample preparations. At least 20 million clean reads of sequencing depth were obtained for each sample. Differential expression analysis of two groups (two biological replicates per condition) was performed using the DESeq R package (1.10.1). DESeq provide statistical routines for determining differential expression in digital gene expression data using a model based on the negative binomial distribution. The resulting P-values were adjusted using the Benjamini and Hochberg's approach for controlling the false discovery rate. Genes with an adjusted P-value $<0.05$ found by DESeq were assigned as differentially expressed. DEGs were defined as genes with FDR less than 0.01 and $\log 2$ fold change larger than 1 (upregulation) or smaller than -1 (downregulation). Gene Ontology (GO) and pathway annotation and enrichment analyses were based on the NCBI COG (https://www.ncbi. nlm.nih.gov/COG/), Gene Ontology Database (http://www.geneonto logy.org/) and KEGG pathway database (http://www.genome.jp/ke $\mathrm{gg} /$ ), respectively. The software Cluster and Java Treeview were used for hierarchical cluster analysis of gene expression patterns.

Adeno-associated virus induced overexpression of human alpha-synuclein. Recombinant AAVs (serotype 2 genome packaged in serotype 9 capsid) were used for the expression of human wild-type $\alpha$-syn, GFP driven by the human synapsin-1 promoter and enhanced using a woodchuck hepatitis virus posttranscriptional regulatory element (WPRE) [50,79].

Stereotaxic injection. Vector solution was bilaterally injected within the SNc region using a $0.2 \mathrm{~mm}$-gauge stainless steel injector connected to a $5 \mu$ l Hamilton syringe. In all experimental groups, the AAV was injected in a volume of $1 \mu \mathrm{l} / \mathrm{side}$ at a rate of $0.2 \mu \mathrm{l} / \mathrm{min}$. The stereotaxic coordinates used (flat skull position) were: $\mathrm{AP}=-3.2 \mathrm{~mm}$; $\mathrm{ML}= \pm 1.2 \mathrm{~mm}, \mathrm{DV}=-4.6 \mathrm{~mm}$ relative to the bregma, according to the atlas of Paxinos and Franklin (2001). Only animals with correct injection placements, verified by analysing immunofluorescence staining of consecutive coronal brain sections, were included in the statistical analysis transgene expression of human wild type alpha-synuclein.

Sample collection and tissue preparation. For collecting feces, mice were placed individually in empty autoclaved cages and allowed to defecate freely in the morning after the day of last treatment. Once feces were formed of each mouse, they were collected immediately in individual sterile EP tubes into liquid nitrogen and then stored at $-80{ }^{\circ} \mathrm{C}$ until next usage.

Statistical analysis. Data are expressed as the mean \pm s.e.m. with at least three biologically independent experiments. Representative morphological images were taken from at least three biologically independent experiments with similar results. Statistical significance was determined using an unpaired two-tailed Student t-test or a two-way ANOVAs followed by Tukey's multiple comparisons test were used. All data were analyzed using the appropriate statistical analysis methods, as specified in the figure legends, with the SPSS software (version 19.0). The test was performed using SPSS. Significance was accepted at *P< $0.05, * * P<0.01$ or $* * * P<0.001$.

\section{Ethics approval and consent to participate}

Not applicable. 


\section{Consent for publication}

Not applicable.

\section{Availability of data and materials}

The datasets used and/or analyzed during the current study are included in this article and its supplementary information files. The datasets are available from the corresponding author on reasonable request.

\section{Funding}

This work was supported by grants from the National Key Research and Development Program of China (2017YFC1700402 to R.Z.), the National Natural Science Foundation of China (No.81471064, No. 81670779 and No. 81870590 to R.Z), the Beijing Municipal Natural Science Foundation (No. 7162097 and No. H2018206641 to R.Z), and the Peking University Research Foundation (No. BMU20140366 to R.Z).

\section{Author contribution}

C.Z. and M.Z. did the experiment, analyzed the data. B.W., J.S., and B.G. participated in experiments. M.Z. and R.Z. wrote the manuscript. R. Z. conceived the idea of study and manuscript writing. All authors reviewed and approved the manuscript for submission.

\section{Declaration of competing interest}

The authors declare no competing financial interests.

\section{Acknowledgement}

We thank Siwang Yu (School of Pharmaceutical Sciences, Peking University Health Science Center) for providing Nrf2-KO mice; Han Xiao (Peking University Third Hospital) for providing NLRP3 KO mice; Changtao Jiang (Peking University Health Science Center) for providing Caspase-1-KO mice. We thank K. Wang (Histology Facility of Department of Anatomy, Histology and Embryology, Peking University) for technical support. We also thank S. Zhu (Department of Physiology and Pathophysiology, Peking University) and M. Ye (Peking University School of Pharmaceutical Sciences) for providing access to necessary equipments.

\section{Abbreviations}

AAV Adeno-associated virus

MPTP 1-methyl-4-phenyl-1,2,3,6-tetrahydropyridine;

GFAP glial fibrillary acidic protein

ASC Apoptosis-associated speck-like protein containing a CARD

Nrf2 Nuclear factor (erythroid-derived 2)-like 2

Iba-1 Ionized calciumbinding adapter molecule 1

IL-4 Interleukin-4

NFKB Nuclear factor kappa-light-chainenhancer of activated B cells

NLRP3 NOD- LRR- and pyrin domaincontaining 3

PD Parkinson's disease

RNAseq RNA sequencing

SNc Substantia nigra pars compacta

TH Tyrosine hydroxylase

DAT Dopamine transporter

TNF- $\alpha$ Tumor necrosis factor- $\alpha$;

$\alpha$-syn Alpha-synuclein

\section{Appendix B. Supplementary data}

Supplementary data related to this article can be found at https:// doi.org/10.1016/j.redox.2021.102134.

\section{References}

[1] E.K. Tan, et al., Parkinson disease and the immune system - associations, mechanisms and therapeutics, Nat. Rev. Neurol. (2020), https://doi.org/10.1038/ s41582-020-0344-4.

[2] R. Gordon, et al., Inflammasome inhibition prevents alpha-synuclein pathology and dopaminergic neurodegeneration in mice, Sci. Transl. Med. 10 (2018), https://doi. org/10.1126/scitranslmed.aah4066.

[3] D. Matheoud, et al., Intestinal infection triggers Parkinson's disease-like symptoms in Pink1(-/-) mice, Nature 571 (2019) 565-569, https://doi.org/10.1038/s41586019-1405-y.

[4] M. Shahnawaz, et al., Discriminating alpha-synuclein strains in Parkinson's disease and multiple system atrophy, Nature 578 (2020) 273-277, https://doi.org/ 10.1038/s41586-020-1984-7.

[5] L. Klingelhoefer, H. Reichmann, Pathogenesis of Parkinson disease-the gut-brain axis and environmental factors, Nat. Rev. Neurol. 11 (2015) 625-636, https://doi. org/10.1038/nrneurol.2015.197.

[6] M.E. Johnson, B. Stecher, V. Labrie, L. Brundin, P. Brundin, Triggers, facilitators, and aggravators: redefining Parkinson's disease pathogenesis, Trends Neurosci. 42 (2019) 4-13, https://doi.org/10.1016/j.tins.2018.09.007.

[7] R.A. Barker, T. consortium, Designing stem-cell-based dopamine cell replacement trials for Parkinson's disease, Nat. Med. 25 (2019) 1045-1053, https://doi.org/ 10.1038/s41591-019-0507-2.

[8] C.V.M. Verschuur, et al., Randomized delayed-start trial of levodopa in Parkinson's disease, N. Engl. J. Med. 380 (2019) 315-324, https://doi.org/10.1056/ NEJMoa1809983.

[9] V.L. Dawson, T.M. Dawson, Promising disease-modifying therapies for Parkinson's disease, Sci. Transl. Med. 11 (2019), https://doi.org/10.1126/scitranslmed, aba1659.

[10] T.M. Axelsen, D.P.D. Woldbye, Gene therapy for Parkinson's disease, an update, J. Parkinsons Dis. 8 (2018) 195-215, https://doi.org/10.3233/JPD-181331.

[11] R.T. Bartus, M.S. Weinberg, R.J. Samulski, Parkinson's disease gene therapy: success by design meets failure by efficacy, Mol. Ther. 22 (2014) 487-497, https:// doi.org/10.1038/mt.2013.281.

[12] S.N. Rai, et al., Anti-inflammatory activity of ursolic acid in MPTP-induced parkinsonian mouse model, Neurotox. Res. 36 (2019) 452-462, https://doi.org/ 10.1007/s12640-019-00038-6.

[13] M. Perni, et al., A natural product inhibits the initiation of alpha-synuclein aggregation and suppresses its toxicity, Proc. Natl. Acad. Sci. U. S. A. 114 (2017) E1009-E1017, https://doi.org/10.1073/pnas.1610586114.

[14] S.W. Mok, et al., Natural products-based polypharmacological modulation of the peripheral immune system for the treatment of neuropsychiatric disorders, Pharmacol. Ther. 208 (2020), 107480, https://doi.org/10.1016/j. pharmthera.2020.107480.

[15] Y.N. Deng, J. Shi, J. Liu, Q.M. Qu, Celastrol protects human neuroblastoma SHSY5Y cells from rotenone-induced injury through induction of autophagy, Neurochem. Int. 63 (2013) 1-9, https://doi.org/10.1016/j.neuint.2013.04.005.

[16] M. Jiang, et al., Celastrol treatment protects against acute ischemic stroke-induced brain injury by promoting an IL-33/ST2 axis-mediated microglia/macrophage M2 polarization, J. Neuroinflammation 15 (2018) 78, https://doi.org/10.1186/ s12974-018-1124-6.

[17] M. Bian, et al., Celastrol protects mouse retinas from bright light-induced degeneration through inhibition of oxidative stress and inflammation, J. Neuroinflammation 13 (2016) 50, https://doi.org/10.1186/s12974-016-0516-8.

[18] C. Cleren, N.Y. Calingasan, J. Chen, M.F. Beal, Celastrol protects against MPTPand 3-nitropropionic acid-induced neurotoxicity, J. Neurochem. 94 (2005) 995-1004, https://doi.org/10.1111/j.1471-4159.2005.03253.x.

[19] D. Paris, et al., Reduction of beta-amyloid pathology by celastrol in a transgenic mouse model of Alzheimer's disease, J. Neuroinflammation 7 (2010) 17, https:// doi.org/10.1186/1742-2094-7-17.

[20] K. Veerappan, S. Natarajan, P. Ethiraj, U. Vetrivel, S. Samuel, Inhibition of IKKbeta by celastrol and its analogues - an in silico and in vitro approach, Pharm. Biol. 55 (2017) 368-373, https://doi.org/10.1080/13880209.2016.1241809.

[21] M.H. Bakar, M.R. Sarmidi, C.K. Kai, H.Z. Huri, H. Yaakob, Amelioration of mitochondrial dysfunction-induced insulin resistance in differentiated 3T3-L1 adipocytes via inhibition of NF-kappaB pathways, Int. J. Mol. Sci. 15 (2014) 22227-22257, https://doi.org/10.3390/ijms151222227.

[22] H. Sassa, Y. Takaishi, H. Terada, The triterpene celastrol as a very potent inhibitor of lipid peroxidation in mitochondria, Biochem. Biophys. Res. Commun. 172 (1990) 890-897, https://doi.org/10.1016/0006-291x(90)90759-g.

[23] E. Angelopoulou, E.S. Pyrgelis, C. Piperi, Neuroprotective potential of chrysin in Parkinson's disease: molecular mechanisms and clinical implications, Neurochem. Int. 132 (2020), 104612, https://doi.org/10.1016/j.neuint.2019.104612.

[24] R.A. Linker, et al., Fumaric acid esters exert neuroprotective effects in neuroinflammation via activation of the Nrf2 antioxidant pathway, Brain 134 (2011) 678-692, https://doi.org/10.1093/brain/awq386.

[25] D. Olagnier, et al., Nrf2 negatively regulates STING indicating a link between antiviral sensing and metabolic reprogramming, Nat. Commun. 9 (2018) 3506, https://doi.org/10.1038/s41467-018-05861-7.

[26] I. Buendia, et al., Nrf2-ARE pathway: an emerging target against oxidative stress and neuroinflammation in neurodegenerative diseases, Pharmacol. Ther. 157 (2016) 84-104, https://doi.org/10.1016/j.pharmthera.2015.11.003.

[27] M. von Otter, et al., Genetic associations of Nrf2-encoding NFE2L2 variants with Parkinson's disease - a multicenter study, BMC Med. Genet. 15 (2014) 131, https:// doi.org/10.1186/s12881-014-0131-4. 
[28] M. von Otter, et al., Association of Nrf2-encoding NFE2L2 haplotypes with Parkinson's disease, BMC Med. Genet. 11 (2010) 36, https://doi.org/10.1186/ 1471-2350-11-36.

[29] H. Xiao, et al., Deprenyl prevents MPP(+)-induced oxidative damage in PC12 cells by the upregulation of Nrf2-mediated NQO1 expression through the activation of PI3K/Akt and Erk, Toxicology 290 (2011) 286-294, https://doi.org/10.1016/j. tox.2011.10.007.

[30] L. Gan, M.R. Vargas, D.A. Johnson, J.A. Johnson, Astrocyte-specific overexpression of Nrf2 delays motor pathology and synuclein aggregation throughout the CNS in the alpha-synuclein mutant (A53T) mouse model, J. Neurosci. 32 (2012) 17775-17787, https://doi.org/10.1523/JNEUROSCI.3049-12.2012.

[31] D. Luo, et al., Natural product celastrol suppressed macrophage M1 polarization against inflammation in diet-induced obese mice via regulating Nrf2/HO-1, MAP kinase and NF-kappaB pathways, Aging (N Y) 9 (2017) 2069-2082, https://doi. org/10.18632/aging.101302.

[32] M.E. Haque, et al., Targeting the microglial NLRP3 inflammasome and its role in Parkinson's disease, Mov. Disord. (2019), https://doi.org/10.1002/mds.27874.

[33] Y. Yan, et al., Dopamine controls systemic inflammation through inhibition of NLRP3 inflammasome, Cell 160 (2015) 62-73, https://doi.org/10.1016/j. cell.2014.11.047.

[34] K.M. von Herrmann, et al., NLRP3 expression in mesencephalic neurons and characterization of a rare NLRP3 polymorphism associated with decreased risk of Parkinson's disease, NPJ Parkinsons Dis 4 (2018) 24, https://doi.org/10.1038/ s41531-018-0061-5.

[35] S. Wang, Y.H. Yuan, N.H. Chen, H.B. Wang, The mechanisms of NLRP3 inflammasome/pyroptosis activation and their role in Parkinson's disease, Int. Immunopharm. 67 (2019) 458-464, https://doi.org/10.1016/j. intimp.2018.12.019.

[36] X. Han, et al., Small molecule-driven NLRP3 inflammation inhibition via interplay between ubiquitination and autophagy: implications for Parkinson disease, Autophagy 15 (2019) 1860-1881, https://doi.org/10.1080/ 15548627.2019.1596481.

[37] E. Lee, et al., MPTP-driven NLRP3 inflammasome activation in microglia plays a central role in dopaminergic neurodegeneration, Cell Death Differ. 26 (2019) 213-228, https://doi.org/10.1038/s41418-018-0124-5.

[38] L.L. Xu, et al., 5-(3,4-Difluorophenyl)-3-(6-methylpyridin-3-yl)-1,2,4-oxadiazole (DDO-7263), a novel Nrf2 activator targeting brain tissue, protects against MPTPinduced subacute Parkinson's disease in mice by inhibiting the NLRP3 inflammasome and protects PC12 cells against oxidative stress, Free Radic. Biol. Med. 134 (2019) 288-303, https://doi.org/10.1016/j. freeradbiomed.2019.01.003.

[39] C. Yang, et al., Astragaloside IV ameliorates motor deficits and dopaminergic neuron degeneration via inhibiting neuroinflammation and oxidative stress in a Parkinson's disease mouse model, Int. Immunopharm. 75 (2019), 105651, https:// doi.org/10.1016/j.intimp.2019.05.036.

[40] W. Xin, Q. Wang, D. Zhang, C. Wang, A new mechanism of inhibition of IL-1beta secretion by celastrol through the NLRP3 inflammasome pathway, Eur. J. Pharmacol. 814 (2017) 240-247, https://doi.org/10.1016/j.ejphar.2017.08.036.

[41] D. Jantas, et al., The extent of neurodegeneration and neuroprotection in two chemical in vitro models related to Parkinson's disease is critically dependent on cell culture conditions, Neurotox. Res. 24 (2013) 41-54, https://doi.org/10.1007/ s12640-012-9374-z.

[42] M. Ahuja, et al., Distinct Nrf2 signaling mechanisms of fumaric acid esters and their role in neuroprotection against 1-methyl-4-phenyl-1,2,3,6-tetrahydropyridineinduced experimental Parkinson's-like disease, J. Neurosci. 36 (2016) 6332-6351, https://doi.org/10.1523/JNEUROSCI.0426-16.2016.

[43] P.C. Chen, et al., Nrf2-mediated neuroprotection in the MPTP mouse model of Parkinson's disease: critical role for the astrocyte, Proc. Natl. Acad. Sci. U. S. A. 106 (2009) 2933-2938, https://doi.org/10.1073/pnas.0813361106.

[44] I. Lastres-Becker, et al., Repurposing the NRF2 activator dimethyl fumarate as therapy against synucleinopathy in Parkinson's disease, Antioxidants Redox Signal. 25 (2016) 61-77, https://doi.org/10.1089/ars.2015.6549.

[45] M.G. Jo, et al., Gintonin mitigates MPTP-induced loss of nigrostriatal dopaminergic neurons and accumulation of alpha-synuclein via the Nrf2/HO-1 pathway, Mol. Neurobiol. 56 (2019) 39-55, https://doi.org/10.1007/s12035-018-1020-1.

[46] Y. Chen, et al., NLRP3 inflammasome pathway is involved in olfactory bulb pathological alteration induced by MPTP, Acta Pharmacol. Sin. 40 (2019) 991-998, https://doi.org/10.1038/s41401-018-0209-1.

[47] C. Qiao, et al., Caspase-1 deficiency alleviates dopaminergic neuronal death via inhibiting caspase-7/AIF pathway in MPTP/p mouse model of Parkinson's disease, Mol. Neurobiol. 54 (2017) 4292-4302, https://doi.org/10.1007/s12035-0169980-5.

[48] Y. Zhou, et al., MicroRNA-7 targets Nod-like receptor protein 3 inflammasome to modulate neuroinflammation in the pathogenesis of Parkinson's disease, Mol. Neurodegener. 11 (2016) 28, https://doi.org/10.1186/s13024-016-0094-3.

[49] J. Cheng, et al., Microglial autophagy defect causes Parkinson disease-like symptoms by accelerating inflammasome activation in mice, Autophagy (2020) 1-13, https://doi.org/10.1080/15548627.2020.1719723.

[50] N. Giordano, et al., Motor learning and metaplasticity in striatal neurons: relevance for Parkinson's disease, Brain 141 (2018) 505-520, https://doi.org/10.1093/ brain/awx351.
[51] L. Cheng, et al., Gene dysregulation is restored in the Parkinson's disease MPTP neurotoxic mice model upon treatment of the therapeutic drug $\mathrm{Cu}(\mathrm{II})(\mathrm{atsm}), \mathrm{Sci}$. Rep. 6 (2016), 22398, https://doi.org/10.1038/srep22398.

[52] L. Fresard, et al., Identification of rare-disease genes using blood transcriptome sequencing and large control cohorts, Nat. Med. 25 (2019) 911-919, https://doi. org/10.1038/s41591-019-0457-8.

[53] L. Fao, S.I. Mota, A.C. Rego, Shaping the Nrf2-ARE-related pathways in Alzheimer's and Parkinson's diseases, Ageing Res. Rev. 54 (2019), 100942, https://doi.org/10.1016/j.arr.2019.100942.

[54] L. Zhang, et al., Fucoidan protects dopaminergic neurons by enhancing the mitochondrial function in a rotenone-induced rat model of Parkinson's disease, Aging Dis 9 (2018) 590-604, https://doi.org/10.14336/AD.2017.0831.

[55] J.R. Liddell, Are astrocytes the predominant cell type for activation of Nrf2 in aging and neurodegeneration? Antioxidants 6 (2017) https://doi.org/10.3390/ antiox6030065.

[56] M.V. Sofroniew, Astrocyte barriers to neurotoxic inflammation, Nat. Rev. Neurosci. 16 (2015) 249-263, https://doi.org/10.1038/nrn3898.

[57] S. Hickman, S. Izzy, P. Sen, L. Morsett, El Khoury, J. Microglia in neurodegeneration, Nat. Neurosci. 21 (2018) 1359-1369, https://doi.org/ 10.1038/s41593-018-0242-x.

[58] D. Charvin, R. Medori, R.A. Hauser, O. Rascol, Therapeutic strategies for Parkinson disease: beyond dopaminergic drugs, Nat. Rev. Drug Discov. 17 (2018) 844, https://doi.org/10.1038/nrd.2018.184.

[59] H. Guo, J.B. Callaway, J.P. Ting, Inflammasomes: mechanism of action, role in disease, and therapeutics, Nat. Med. 21 (2015) 677-687, https://doi.org/10.1038/ nm.3893.

[60] M.T. Heneka, et al., NLRP3 is activated in Alzheimer's disease and contributes to pathology in APP/PS1 mice, Nature 493 (2013) 674-678, https://doi.org/ 10.1038/nature11729.

[61] Z. Mao, et al., The NLRP3 inflammasome is involved in the pathogenesis of Parkinson's disease in rats, Neurochem. Res. 42 (2017) 1104-1115, https://doi. org/10.1007/s11064-017-2185-0.

[62] A.O. Pires, et al., Old and new challenges in Parkinson's disease therapeutics, Prog. Neurobiol. 156 (2017) 69-89, https://doi.org/10.1016/j.pneurobio.2017.04.006.

[63] J.B. Koprich, L.V. Kalia, J.M. Brotchie, Animal models of alpha-synucleinopathy for Parkinson disease drug development, Nat. Rev. Neurosci. 18 (2017) 515-529, https://doi.org/10.1038/nrn.2017.75.

[64] A. Elkouzi, V. Vedam-Mai, R.S. Eisinger, M.S. Okun, Emerging therapies in Parkinson disease - repurposed drugs and new approaches, Nat. Rev. Neurol. 15 (2019) 204-223, https://doi.org/10.1038/s41582-019-0155-7.

[65] A. Lau, et al., alpha-Synuclein strains target distinct brain regions and cell types, Nat. Neurosci. 23 (2020) 21-31, https://doi.org/10.1038/s41593-019-0541-x.

[66] P. Jiang, D.W. Dickson, Parkinson's disease: experimental models and reality, Acta Neuropathol. 135 (2018) 13-32, https://doi.org/10.1007/s00401-017-1788-5.

[67] R. Cascao, J.E. Fonseca, L.F. Moita, Celastrol: a spectrum of treatment opportunities in chronic diseases, Front. Med. 4 (2017) 69, https://doi.org/ 10.3389/fmed.2017.00069.

[68] T.W. Mu, et al., Chemical and biological approaches synergize to ameliorate protein-folding diseases, Cell 134 (2008) 769-781, https://doi.org/10.1016/j. cell.2008.06.037.

[69] J. Lee, et al., Withaferin A is a leptin sensitizer with strong antidiabetic properties in mice, Nat. Med. 22 (2016) 1023-1032, https://doi.org/10.1038/nm.4145.

[70] Zhao, M. et al. The DJ1-Nrf2-STING axis mediates the neuroprotective effects of Withaferin A in Parkinson's disease. Cell Death Differ. 28, 2517-2535, doi: 10.1038/s41418-021-00767-2(2021).

[71] J.Z. Xin, et al., alpha1-AR overactivation induces cardiac inflammation through NLRP3 inflammasome activation, Acta Pharmacol. Sin. 41 (2020) 311-318, https://doi.org/10.1038/s41401-019-0305-x.

[72] S.Y. Zhang, et al., Adipocyte-derived lysophosphatidylcholine activates adipocyte and adipose tissue macrophage nod-like receptor protein 3 inflammasomes mediating homocysteine-induced insulin resistance, EBioMedicine 31 (2018) 202-216, https://doi.org/10.1016/j.ebiom.2018.04.022.

[73] V. Jackson-Lewis, S. Przedborski, Protocol for the MPTP mouse model of Parkinson's disease, Nat. Protoc. 2 (2007) 141-151, https://doi.org/10.1038/ nprot.2006.342.

[74] T.I. Kam, et al., Poly(ADP-ribose) drives pathologic alpha-synuclein neurodegeneration in Parkinson's disease, Science 362 (2018), https://doi.org/ 10.1126/science.aat8407.

[75] R. Zheng, et al., Deficiency of the RII $\beta$ subunit of PKA affects locomotor activity and energy homeostasis in distinct neuronal populations, Proc. Natl. Acad. Sci. U. S. A. 110 (2013) E1631-E1640, https://doi.org/10.1073/pnas.1219542110.

[76] S.P. Yun, et al., Block of A1 astrocyte conversion by microglia is neuroprotective in models of Parkinson's disease, Nat. Med. 24 (2018) 931-938, https://doi.org/ 10.1038/s41591-018-0051-5.

[77] P.T. Tsai, et al., Autistic-like behaviour and cerebellar dysfunction in Purkinje cell Tsc1 mutant mice, Nature 488 (2012) 647-651, https://doi.org/10.1038/ nature11310.

[78] T.R. Sampson, et al., Gut microbiota regulate motor deficits and neuroinflammation in a model of Parkinson's disease, Cell 167 (2016) 1469-1480, https://doi.org/10.1016/j.cell.2016.11.018, e1412.

[79] J.H. Nam, et al., TRPV1 on astrocytes rescues nigral dopamine neurons in Parkinson's disease via CNTF, Brain 138 (2015) 3610-3622, https://doi.org/ 10.1093/brain/awv297. 